\title{
Flavor changing in the flipped trinification
}

\author{
D. N. Dinh, ${ }^{1}$ D. T. Huong, ${ }^{1}$ N. T. Duy, ${ }^{2}$ N. T. Nhuan, ${ }^{2}$ L. D. Thien, ${ }^{2}$ and Phung Van Dong ${ }^{3,4, *}$ \\ ${ }^{1}$ Institute of Physics, Vietnam Academy of Science and Technology, \\ 10 Dao Tan, Ba Dinh, Hanoi 100000, Vietnam \\ ${ }^{2}$ Graduate University of Science and Technology, Vietnam Academy of Science and Technology, \\ 18 Hoang Quoc Viet, Cau Giay, Hanoi 100000, Vietnam \\ ${ }^{3}$ Phenikaa Institute for Advanced Study, Phenikaa University, Hanoi 100000, Vietnam \\ ${ }^{4}$ Faculty of Basic Science and Faculty of Materials Science and Engineering, Phenikaa University, \\ Hanoi 100000, Vietnam
}

(Received 23 January 2019; published 8 March 2019)

\begin{abstract}
The flipped trinification, a framework for unifying the 3-3-1 and left-right symmetries, has recently been proposed in order to solve profound questions, the weak parity violation and the number of families, besides the implication for neutrino mass generation and dark matter stability. In this work, we argue that this gauge completion naturally provides flavor-changing neutral currents in both quark and lepton sectors. The quark flavor changing happens at the tree level due to the nonuniversal couplings of $Z_{L, R}^{\prime}$, while the lepton flavor changing $l \rightarrow l^{\prime} \gamma$ starts from the one-loop level, which is significantly contributed by the new charged currents of $Y_{L, R}$ that couple ordinary leptons to exotic leptons. These effects disappear in the minimal left-right model but are present in the framework characterizing a flipped trinification symmetry.
\end{abstract}

DOI: 10.1103/PhysRevD.99.055005

\section{INTRODUCTION}

The experiments of neutrino oscillations caused by nonzero small neutrino masses and flavor mixing have provided the most important evidence that proves the new physics beyond the standard model [1]. The compelling way to address the neutrino masses is to introduce right-handed neutrinos into the standard model, by which the neutrino mass generation is done by seesaw mechanisms [2]. The pioneering model that recognizes the seesaw mechanisms is the minimal left-right symmetric model [3], in which the neutrino masses were predicted before the experimental confirmations.

The minimal left-right symmetric model offers the possibility to understand the origin of the parity violation of weak interactions, but as the standard model, it neither shows why there are only three fermion generations nor addresses dark matter stability that accounts for more than $25 \%$ mass-energy density of the Universe [4]. Indeed, the lightest right-handed neutrino may have a kilo-electron-volt mass responsible for warm dark matter, but it would

\footnotetext{
* Corresponding author. dong.phungvan@phenikaa-uni.edu.vn

Published by the American Physical Society under the terms of the Creative Commons Attribution 4.0 International license. Further distribution of this work must maintain attribution to the author(s) and the published article's title, journal citation, and DOI. Funded by SCOAP.
}

overpopulate the Universe due to gauge interactions, which require nonstandard dilution mechanisms [5]. On the other hand, the cold dark matter scenario that adds a new field as well as imposes a stabilizing symmetry is arbitrary, ad hoc included [6].

It is well established that the 3-3-1 model [7] provides a potential solution to the generation number and addresses the issue of dark matter naturally [8]. Hence, we have recently proposed a theoretical model that unifies both the left-right and 3-3-1 symmetries, resulting in a $S U(3)_{C} \otimes$ $S U(3)_{L} \otimes S U(3)_{R} \otimes U(1)_{X}$ gauge group, called flipped trinification [9] (for other interpretations, see Ref. [10]). This model inherits all the nice features of both left-right and 3-3-1 models. Particularly, dark matter naturally exists and along with normal matter forms gauge multiplets by the gauge symmetry, whereas the three generations emerge as a result of anomaly cancellation. Moreover, the origin of the matter parity and the dark matter stability are determined by a residual gauge symmetry. The new physics predicted occurs at tera-electron-volt scale, giving rise to interesting signatures at current colliders.

An intriguing feature of the flipped trinification is that flavor-violating interactions appear in both quark and lepton sectors. As a trinification symmetry is flipped, both left- and right-handed quark flavors transform differently under $S U(3)_{L, R}$. Consequently, they lead to tree-level flavor-changing neutral currents (FCNCs) that couple to $Z_{L, R}^{\prime}$, and the relevant observables after integrating out $Z_{L, R}^{\prime}$ 
depend on both left- and right-handed quark mixing matrices. Further, the discovery of neutrino oscillations suggests lepton flavor violation (LFV), but the charged LFV has never been observed. As the minimal left-right gauge symmetry is enlarged to trinification, the model predicts new non-Hermitian gauge bosons $Y_{L, R}$ that couple charged leptons to new heavy leptons. This is the main source for charged LFV processes $l \rightarrow l^{\prime} \gamma$ that are mediated by $Y_{L, R}$ in one-loop corrections, since the new leptons mix. Additionally, the contributions of $W_{L, R}$ due to the neutrino mixing and of new Higgs bosons to such charged LFV processes will be taken into account. Moreover, the flipped trinification has scalar fields that couple both charged leptons and flavor change. This leads to tree-level charged LFV processes such as $\tau \rightarrow 3 \mu(3 e), \mu \rightarrow 3 e$, and so forth.

Let us recall that, due to the left-right symmetry, the model requires a bitriplet $\varphi$, two triplets $\chi_{L}$ and $\chi_{R}$, and two sextets $\sigma_{L}$ and $\sigma_{R}$, which provide the gauge symmetry breaking and mass generation. Since $\chi_{L}$ and $\sigma_{L}$ have small vacuum expectation values (VEVs), their roles were ignored in the previous study [9]. In this work, we will turn on their effects when including the full scalar sector, which contributes to the gauge symmetry breaking pattern and mass spectra. We show that the VEVs of $\chi_{L}$ and $\sigma_{L}$ do not significantly affect the gauge boson masses, but the neutrino and Higgs spectra are modified.

The rest of this paper is organized as follows. In Sec. II, we reconsider the model with the complete scalar content. In Sec. III, we study the tree-level FCNCs and their contributions to neutral meson mixings, which are mediated by new gauge bosons $Z_{L}^{\prime}$ and $Z_{R}^{\prime}$. In Sec. IV, we present analytic expressions and numerical results for the specific charged LFV processes. Finally, we conclude this work in Sec. V.

\section{REVIEW OF THE MODEL}

This section necessarily imposes $\chi_{L}$ and $\sigma_{L}$ due to the left-right symmetry, which were omitted in the previous study for mathematical simplicity [9].

\section{A. Symmetry and field content}

Left-right symmetrizing the 3-3-1 group [7], we obtain a gauge symmetry,

$$
S U(3)_{C} \otimes S U(3)_{L} \otimes S U(3)_{R} \otimes U(1)_{X},
$$

which matches a flipped trinification and preserves the $S U(3)_{L}$ and $S U(3)_{R}$ interchange. The electric charge operator is given by

$$
Q=T_{3 L}+T_{3 R}+\beta\left(T_{8 L}+T_{8 R}\right)+X,
$$

where $T_{i L, R}(i=1,2,3, \ldots, 8)$ and $X$ are $S U(3)_{L, R}$ and $U(1)_{X}$ charges, respectively. The baryon minus lepton number is identified as

$$
\frac{1}{2}(B-L)=\beta\left(T_{8 L}+T_{8 R}\right)+X,
$$

which is noncommutative, in contrast to the usual (Abelian) extensions. We further define a basic electric charge as $q=-(1+\sqrt{3} \beta) / 2$.

Analogously, the fermion content is obtained from those of the 3-3-1 model by left-right symmetrization, which yields

$$
\begin{aligned}
& \psi_{a L}=\left(\begin{array}{c}
\nu_{a L} \\
e_{a L} \\
N_{a L}^{q}
\end{array}\right) \sim\left(1,3,1, \frac{q-1}{3}\right), \\
& \psi_{a R}=\left(\begin{array}{c}
\nu_{a R} \\
e_{a R} \\
N_{a R}^{q}
\end{array}\right) \sim\left(1,1,3, \frac{q-1}{3}\right), \\
& Q_{\alpha L}=\left(\begin{array}{c}
d_{\alpha L} \\
-u_{\alpha L} \\
J_{\alpha L}^{-q-\frac{1}{3}}
\end{array}\right) \sim\left(3,3^{*}, 1,-\frac{q}{3}\right), \\
& Q_{\alpha R}=\left(\begin{array}{c}
d_{\alpha R} \\
-u_{\alpha R} \\
J_{\alpha R}^{-q-\frac{1}{3}}
\end{array}\right) \sim\left(3,1,3^{*},-\frac{q}{3}\right),
\end{aligned}
$$

$$
\begin{gathered}
Q_{3 L}=\left(\begin{array}{c}
u_{3 L} \\
d_{3 L} \\
J_{3 L}^{q+\frac{2}{3}}
\end{array}\right) \sim\left(3,3,1, \frac{q+1}{3}\right), \\
Q_{3 R}=\left(\begin{array}{c}
u_{3 R} \\
d_{3 R} \\
J_{3 R}^{q+\frac{2}{3}}
\end{array}\right) \sim\left(3,1,3, \frac{q+1}{3}\right),
\end{gathered}
$$

where $a=1,2,3$ and $\alpha=1,2$ are generation indices. The model predicts new fermions $N_{a}, J_{a}$, besides the righthanded neutrinos $\nu_{a R}$. The fermion sector is more economical than that of the well-known trinification [11]. In contrast to the trinification, the $S U(3)_{L}$ or $S U(3)_{R}$ anomaly cancellation requires the number of generations to match that of colors and the third quark generation to transform under $S U(3)_{L, R}$ differently from the first two quark generations, analogous to the 3-3-1 model [7].

To break the gauge symmetry and generate the masses appropriately, the scalar multiplets are supplied as 


$$
\begin{aligned}
\phi & =\left(\begin{array}{lll}
\phi_{11}^{0} & \phi_{12}^{+} & \phi_{13}^{-q} \\
\phi_{21}^{-} & \phi_{22}^{0} & \phi_{23}^{-1-q} \\
\phi_{31}^{q} & \phi_{32}^{1+q} & \phi_{33}^{0}
\end{array}\right) \sim\left(1,3,3^{*}, 0\right), \\
\chi_{L}= & \left(\begin{array}{c}
\chi_{1}^{-q} \\
\chi_{2}^{-q-1} \\
\chi_{3}^{0}
\end{array}\right)_{L} \sim\left(1,3,1,-\frac{2 q+1}{3}\right), \\
\chi_{R}= & \left(\begin{array}{c}
\chi_{1}^{-q} \\
\chi_{2}^{-q-1} \\
\chi_{3}^{0}
\end{array}\right)_{R} \sim\left(1,1,3,-\frac{2 q+1}{3}\right), \\
\sigma_{L}= & \left(\begin{array}{ccc}
\sigma_{11}^{0} & \frac{\sigma_{12}^{-}}{\sqrt{2}} & \frac{\sigma_{13}^{q}}{\sqrt{2}} \\
\frac{\sigma_{12}^{-}}{\sqrt{2}} & \sigma_{22}^{--} & \frac{\sigma_{23}^{q-1}}{\sqrt{2}} \\
\frac{\sigma_{13}^{q}}{\sqrt{2}} & \frac{\sigma_{23}^{q-1}}{\sqrt{2}} & \sigma_{33}^{2 q}
\end{array}\right)_{L} \sim\left(1,6,1, \frac{2(q-1)}{3}\right), \\
\sigma_{R}= & \left(\begin{array}{lll}
\sigma_{11}^{0} & \frac{\sigma_{12}^{-}}{\sqrt{2}} & \frac{\sigma_{13}^{q}}{\sqrt{2}} \\
\frac{\sigma_{12}^{-}}{\sqrt{2}} & \sigma_{22}^{--} & \frac{\sigma_{23}^{q-1}}{\sqrt{2}} \\
\frac{\sigma_{13}^{q}}{\sqrt{2}} & \frac{\sigma_{23}^{q-1}}{\sqrt{2}} & \sigma_{33}^{2 q}
\end{array}\right)_{R} \sim\left(1,1,6, \frac{2(q-1)}{3}\right),
\end{aligned}
$$

which reflect the left-right symmetry. The corresponding VEVs are given by

$$
\begin{gathered}
\langle\phi\rangle=\frac{1}{\sqrt{2}}\left(\begin{array}{ccc}
u & 0 & 0 \\
0 & u^{\prime} & 0 \\
0 & 0 & w
\end{array}\right), \\
\left\langle\chi_{L}\right\rangle=\frac{1}{\sqrt{2}}\left(\begin{array}{c}
0 \\
0 \\
w_{L}
\end{array}\right), \quad\left\langle\chi_{R}\right\rangle=\frac{1}{\sqrt{2}}\left(\begin{array}{c}
0 \\
0 \\
w_{R}
\end{array}\right),
\end{gathered}
$$

$$
\begin{aligned}
& \left\langle\sigma_{L}\right\rangle=\frac{1}{\sqrt{2}}\left(\begin{array}{ccc}
\Lambda_{L} & 0 & 0 \\
0 & 0 & 0 \\
0 & 0 & 0
\end{array}\right), \\
& \left\langle\sigma_{R}\right\rangle=\frac{1}{\sqrt{2}}\left(\begin{array}{ccc}
\Lambda_{R} & 0 & 0 \\
0 & 0 & 0 \\
0 & 0 & 0
\end{array}\right) .
\end{aligned}
$$

As shown in Ref. [9], the symmetry breaking proceeds through several schemes, depending on the hierarchy arrangements of the VEVs. All the schemes lead to the existence of a residual discrete gauge symmetry that conserves every VEV, called matter parity,

$$
W_{P}=(-1)^{3(B-L)+2 s}=(-1)^{6\left[\beta\left(T_{8 L}+T_{8 R}\right)+X\right]+2 s} .
$$

This parity ensures the stability of dark matter, which is unified with normal matter in the gauge multiplets (see Ref. [9] for details of the dark sector and dark matter candidates). For consistency, we assume $\Lambda_{R}, w_{R}, w \gg$ $u, u^{\prime} \gg \Lambda_{L}, w_{L}$, appropriate for the potential minimization. (Indeed, the minimization conditions imply $\Lambda_{L} \simeq 0$, $w_{L} \simeq 0$, where the small nonzero values come from abnormal perturbative interactions, as seen in the next section). This means that the flipped trinification is broken down to the standard model and matter parity and then to the remnant $S U(3)_{C} \otimes U(1)_{Q} \otimes W_{P}$, where the left-right asymmetry is explicitly recognized at the electroweak phase due to $w \neq 0, w_{R} \neq w_{L}$, and $\Lambda_{R} \neq \Lambda_{L}$.

\section{B. Fermion masses}

First, we consider the physical states and masses of fermions. They arise from the Yukawa interactions as

$$
\begin{aligned}
\mathcal{L}_{\text {Yukawa }}= & x_{a b}\left(\bar{\psi}_{a R}^{c} \sigma_{R}^{\dagger} \psi_{b R}+\bar{\psi}_{a L}^{c} \sigma_{L}^{\dagger} \psi_{b L}\right)+y_{a b} \bar{\psi}_{a L} \phi \psi_{b R}+\frac{z_{a b}}{M} \bar{\psi}_{a L} \chi_{L} \chi_{R}^{*} \psi_{b R}+k_{33} \bar{Q}_{3 L} \phi Q_{3 R}+k_{\alpha \beta} \bar{Q}_{\alpha L} \phi^{*} Q_{\beta R} \\
& +\frac{k_{33}^{\prime}}{M} \bar{Q}_{3 L} \chi_{L} \chi_{R}^{*} Q_{3 R}+\frac{k_{\alpha \beta}^{\prime}}{M} \bar{Q}_{\alpha L} \chi_{L}^{*} \chi_{R} Q_{\beta R}+\frac{t_{3 \alpha}}{M}\left(\bar{Q}_{3 L} \phi \chi_{R}^{*} Q_{\alpha R}+\bar{Q}_{3 R} \phi^{*} \chi_{L}^{*} Q_{\alpha L}\right) \\
& +\frac{t_{\alpha 3}}{M}\left(\bar{Q}_{\alpha L} \phi^{*} \chi_{R} Q_{3 R}+\bar{Q}_{\alpha R} \phi \chi_{L} Q_{3 L}\right)+\text { H.c. },
\end{aligned}
$$

where $M$ is a new physics scale that defines the effective interactions. The left-right symmetry demands that the couplings $y, z, k$, and $k^{\prime}$ are Hermitian, whereas $x$ and $t$ are generic.

After the symmetry breaking, the Yukawa Lagrangian yields fermion masses. The new leptons get a large mass at the new physical scale as follows:

$$
\mathcal{L}_{\text {mass }}^{N}=\left(\frac{y_{a b}}{\sqrt{2}} w+\frac{z_{a b}}{2 M} w_{L} w_{R}\right) \bar{N}_{a L} N_{b R}+\text { H.c. }
$$

The ordinary charged leptons obtain a mass at the weak scale, 


$$
\mathcal{L}_{\text {mass }}^{l}=\left(\frac{y_{a b}}{\sqrt{2}} u^{\prime}\right) \bar{l}_{a L} l_{b R}+\text { H.c. }
$$

Note that the new leptons do not mix with the ordinary leptons due to the matter parity conservation. If the effective interactions are neglected, they have the same mixing matrices.

The Lagrangian (12) allows neutrinos to have both kinds of mass terms: Dirac and Majorana. In the basis $\left(\nu_{L}, \nu_{R}^{c}\right)$, the neutrino mass matrix is given by

$$
\mathcal{M}_{\nu}=\left(\begin{array}{cc}
M_{\nu}^{L} & M_{\nu}^{D} \\
\left(M_{\nu}^{D}\right)^{T} & M_{\nu}^{R}
\end{array}\right)
$$

where the explicit forms of $M_{\nu}^{D}, M_{\nu}^{L}$, and $M_{\nu}^{R}$ are

$$
\begin{aligned}
& \left(M_{\nu}^{D}\right)_{a b}=-\frac{y_{a b}}{\sqrt{2}} u, \\
& \left(M_{\nu}^{L}\right)_{a b}=-\sqrt{2} x_{a b} \Lambda_{L}, \\
& \left(M_{\nu}^{R}\right)_{a b}=-\sqrt{2} x_{a b} \Lambda_{R} .
\end{aligned}
$$

Because of the condition $\Lambda_{L} \ll u \ll \Lambda_{R}$, the active neutrinos $\left(\sim \nu_{L}\right)$ gain small masses via the seesaw mechanisms,

$$
M_{\nu} \simeq-\sqrt{2} x \Lambda_{L}+\frac{1}{2 \sqrt{2}} y x^{-1} y^{T} \frac{u^{2}}{\Lambda_{R}}
$$

whereas the sterile neutrinos $\left(\sim \nu_{R}\right)$ have large masses at the $\Lambda_{R}$ scale, $M_{\nu}^{\prime} \simeq-\sqrt{2} x \Lambda_{R}$.

The exotic quarks do not mix with ordinary quarks due to the matter parity conservation and have the mass terms given from (12) by

$$
\begin{aligned}
\mathcal{L}_{\text {mass }}^{J}= & \left(\frac{k_{33}}{\sqrt{2}} w+\frac{k_{33}^{\prime}}{2 M} w_{L} w_{R}\right) \bar{J}_{3 L} J_{3 R} \\
& +\left(\frac{k_{\alpha \beta}}{\sqrt{2}} w+\frac{k_{\alpha \beta}^{\prime}}{2 M} w_{L} w_{R}\right) \bar{J}_{\alpha L} J_{\beta R}+\text { H.c. }
\end{aligned}
$$

which are all at the new physics scale.

Denoting $u_{L, R}=\left(u_{1}, u_{2}, u_{2}\right)_{L, R}^{T} \quad$ and $\quad d_{L, R}=\left(d_{1}, d_{2}\right.$, $\left.d_{3}\right)_{L, R}^{T}$, the ordinary quarks achieve mass terms

$$
\mathcal{L}^{u, d}=-\bar{u}_{L} \mathcal{M}^{u} u_{R}-\bar{d}_{L} \mathcal{M}^{d} d_{R}+\text { H.c. },
$$

where

$$
\begin{gathered}
\mathcal{M}^{u}=-\frac{1}{\sqrt{2}}\left(\begin{array}{ccc}
k_{11} u^{\prime} & k_{12} u^{\prime} & \frac{-t_{13}}{M \sqrt{2}} u^{\prime}\left(w_{L}+w_{R}\right) \\
k_{21} u^{\prime} & k_{22} u^{\prime} & \frac{-t_{23}}{M \sqrt{2}} u^{\prime}\left(w_{L}+w_{R}\right) \\
\frac{t_{31}}{M \sqrt{2}} u\left(w_{L}+w_{R}\right) & \frac{t_{32}}{M \sqrt{2}} u\left(w_{L}+w_{R}\right) & k_{33} u
\end{array}\right), \\
\mathcal{M}^{d}=-\frac{1}{\sqrt{2}}\left(\begin{array}{ccc}
k_{11} u & k_{12} u & \frac{-t_{13}}{M \sqrt{2}} u\left(w_{L}+w_{R}\right) \\
k_{21} u & k_{22} u & \frac{-t_{23}}{M \sqrt{2}} u\left(w_{L}+w_{R}\right) \\
\frac{t_{31}}{M \sqrt{2}} u^{\prime}\left(w_{L}+w_{R}\right) & \frac{t_{32}}{M \sqrt{2}} u^{\prime}\left(w_{L}+w_{R}\right) & k_{33} u^{\prime}
\end{array}\right) .
\end{gathered}
$$

Applying biunitary transformations, the mass matrices can be diagonalized as

$$
M^{d}=V_{d L}^{\dagger} \mathcal{M}^{d} V_{d R}, \quad M^{u}=V_{u L}^{\dagger} \mathcal{M}^{u} V_{u R},
$$

where $M^{u}$ and $M^{d}$ are diagonal matrices that consist of respective physical quark masses at the weak scale, given that $M \sim w_{R}$. Note that the mass eigenstates $u^{\prime}=(u, c, t)^{T}$ and $d^{\prime}=(d, s, b)^{T}$ are related to the gauge states by $u_{L, R}=$ $V_{u L, R} u_{L, R}^{\prime}$ and $d_{L, R}=V_{d L, R} d_{L, R}^{\prime}$.

\section{Gauge boson masses}

The presence of the scalar multiplets $\sigma_{L}$ and $\chi_{L}$ does not significantly change the mass spectrum of the gauge bosons that was derived in Ref. [9]. Hereafter, we summarize the main results of the gauge sector. The gauge bosons $W_{L}$ and $W_{R}$ slightly mix, which yields eigenstates

$$
W_{1}=c_{\xi} W_{L}-s_{\xi} W_{R}, \quad W_{2}=s_{\xi} W_{L}+c_{\xi} W_{R},
$$

where the mixing angle $\xi$ is defined by 


$$
t_{2 \xi}=\frac{4 t_{R} u u^{\prime}}{2 \Lambda_{L}^{2}-2 \Lambda_{R}^{2} t_{R}^{2}-\left(t_{R}^{2}-1\right)\left(u^{2}+u^{\prime 2}\right)} \simeq-\frac{2 u u^{\prime}}{t_{R} \Lambda_{R}^{2}} \ll 1 .
$$

The $W_{1,2}$ masses are given by

$$
\begin{gathered}
m_{W_{1}}^{2} \simeq \frac{g_{L}^{2}}{4}\left[u^{2}+u^{\prime 2}+2 \Lambda_{L}^{2}-\frac{4 t_{R}^{2} u^{2} u^{\prime 2}}{\left(t_{R}^{2}-1\right)\left(u^{2}+u^{\prime 2}\right)+2 t_{R}^{2} \Lambda_{R}^{2}-2 \Lambda_{L}^{2}}\right] \simeq \frac{g_{L}^{2}}{4}\left(u^{2}+u^{\prime 2}\right), \\
m_{W_{2}}^{2} \simeq \frac{g_{R}^{2}}{4}\left[u^{2}+u^{\prime 2}+2 \Lambda_{R}^{2}+\frac{4 t_{R}^{2} u^{2} u^{\prime 2}}{\left(t_{R}^{2}-1\right)\left(u^{2}+u^{\prime 2}\right)+2 t_{R}^{2} \Lambda_{R}^{2}-2 \Lambda_{L}^{2}}\right] \simeq \frac{g_{R}^{2}}{2} \Lambda_{R}^{2},
\end{gathered}
$$

where $g_{L}$ and $g_{R}$ are $S U(3)_{L, R}$ couplings, respectively, which match $t_{R} \equiv g_{R} / g_{L}=1$ at the flipped trinification scale due to the left-right symmetry. At the low energy, they may separate, $t_{R} \neq 1$, due to the different contributions to the running couplings. $W_{1}$ is identical to the standard model $W$ boson, implying $u^{2}+u^{\prime 2}=(246 \mathrm{GeV})^{2}$, while $W_{2}$ is new.

Besides, the model predicts new non-Hermitian gauge bosons $X_{L, R}^{ \pm q}$ and $Y_{L, R}^{ \pm(q+1)}$ that couple to the charges $T_{4} \mp i T_{5}$ and $T_{6} \mp i T_{7}$, respectively. The physical states are

$$
\begin{array}{cl}
X_{1}^{ \pm q}=c_{\xi_{1}} X_{L}^{ \pm q}-s_{\xi_{1}} X_{R}^{ \pm q}, & X_{2}^{ \pm q}=s_{\xi_{1}} X_{L}^{ \pm q}+c_{\xi_{1}} X_{R}^{ \pm q}, \\
Y_{1}^{ \pm(1+q)}=c_{\xi_{2}} Y_{L}^{ \pm(1+q)}-s_{\xi_{2}} Y_{R}^{ \pm(1+q)}, & Y_{2}^{ \pm(1+q)}=s_{\xi_{2}} Y_{L}^{ \pm(1+q)}+c_{\xi_{2}} Y_{R}^{ \pm(1+q)} .
\end{array}
$$

Here, the mixing angles $\xi_{1}$ and $\xi_{2}$ are obtained as

$$
\begin{gathered}
t_{2 \xi_{1}}=\frac{4 t_{R} u w}{u^{2}+w^{2}+w_{L}^{2}+2 \Lambda_{L}^{2}-t_{R}^{2}\left(u^{2}+w^{2}+w_{R}^{2}+2 \Lambda_{R}^{2}\right)} \sim \frac{u}{w}, \\
t_{2 \xi_{2}}=\frac{4 t_{R} u^{\prime} w}{u^{\prime 2}+w^{2}+w_{L}^{2}-t_{R}^{2}\left(u^{\prime 2}+w_{R}^{2}+w^{2}\right)} \sim \frac{u^{\prime}}{w} .
\end{gathered}
$$

And the gauge boson masses are given by

$$
\begin{gathered}
m_{X_{1}}^{2}=\frac{g_{L}^{2}}{4}\left[u^{2}+w^{2}+w_{L}^{2}+2 \Lambda_{L}^{2}+\frac{4 t_{R}^{2} u^{2} w^{2}}{u^{2}+w^{2}+w_{L}^{2}+2 \Lambda_{L}^{2}-t_{R}^{2}\left(u^{2}+w^{2}+w_{R}^{2}+2 \Lambda_{R}^{2}\right)}\right] \simeq \frac{g_{L}^{2}}{4} w^{2}, \\
m_{X_{2}}^{2}=\frac{g_{R}^{2}}{4}\left[u^{2}+w^{2}+w_{R}^{2}+2 \Lambda_{R}^{2}-\frac{4 u^{2} w^{2}}{u^{2}+w^{2}+w_{L}^{2}+2 \Lambda_{L}^{2}-t_{R}^{2}\left(u^{2}+w^{2}+w_{R}^{2}+2 \Lambda_{R}^{2}\right)}\right] \simeq \frac{g_{R}^{2}}{4}\left(w^{2}+w_{R}^{2}+2 \Lambda_{R}^{2}\right), \\
m_{Y_{1}}^{2}=\frac{g_{L}^{2}}{4}\left[u^{\prime 2}+w^{2}+w_{L}^{2}+\frac{4 t_{R}^{2} u^{\prime 2} w^{2}}{u^{\prime 2}+w^{2}+w_{L}^{2}-t_{R}^{2}\left(u^{\prime 2}+w^{2}+w_{R}^{2}\right)}\right] \simeq \frac{g_{L}^{2}}{4} w^{2}, \\
m_{Y_{2}}^{2}=\frac{g_{R}^{2}}{4}\left[u^{\prime 2}+w^{2}+w_{R}^{2}-\frac{4 u^{\prime 2} w^{2}}{u^{\prime 2}+w^{2}+w_{L}^{2}-t_{R}^{2}\left(u^{\prime 2}+w^{2}+w_{R}^{2}\right)}\right] \simeq \frac{g_{R}^{2}}{4}\left(w^{2}+w_{R}^{2}\right) .
\end{gathered}
$$

The neutral gauge bosons $A_{3 L, R}, A_{8 L, R}$, and $B$, which couple to the charges $T_{3 L, R}, T_{8 L, R}$, and $X$, respectively, mix via a $5 \times 5$ mass matrix, given in the Appendix. The photon field is

$$
A=s_{W} A_{3 L}+c_{W}\left(\frac{t_{W}}{t_{R}} A_{3 R}+\beta t_{W} A_{8 L}+\beta \frac{t_{W}}{t_{R}} A_{8 R}+\frac{t_{W}}{t_{X}} B\right)
$$

which is massless, where $t_{X} \equiv g_{X} / g_{L}$ is $U(1)_{X} / S U(3)_{L}$ coupling ratio. The sine of the Weinberg angle is $s_{W}=t_{X} t_{R} / \sqrt{t_{X}^{2}\left(1+\beta^{2}\right)+t_{R}^{2}\left(1+t_{X}^{2}\left(1+\beta^{2}\right)\right)}$, obtained by matching the electromagnetic gauge coupling [12]. As usual, the standard model $Z$ boson is given orthogonally to $A$ by 
$Z_{L}=c_{W} A_{3 L}-s_{W}\left(\frac{t_{W}}{t_{R}} A_{3 R}+\beta t_{W} A_{8 L}+\beta \frac{t_{W}}{t_{R}} A_{8 R}+\frac{t_{W}}{t_{X}} B\right)$.

New neutral gauge bosons take the forms that are orthogonal to both $A$ and $Z_{L}$, i.e., to the $U(1)_{Y}$ gauge field in parentheses,

$$
\begin{gathered}
Z_{L}^{\prime}=\varsigma_{1} t_{X} t_{W} \beta A_{3 R}-\frac{t_{W}}{\varsigma_{1} t_{X} t_{R}} A_{8 L}+\varsigma_{1} t_{X} t_{W} \beta^{2} A_{8 R}+\varsigma_{1} t_{R} t_{W} \beta B \\
Z_{R}=-\frac{\varsigma_{1}}{\varsigma} A_{3 R}+\varsigma \varsigma_{1} t_{X}^{2} \beta A_{8 R}+\varsigma \varsigma_{1} t_{X} t_{R} B \\
Z_{R}^{\prime}=\varsigma\left(t_{R} A_{8 R}-t_{X} \beta B\right)
\end{gathered}
$$

where $\varsigma=1 / \sqrt{t_{R}^{2}+\beta^{2} t_{X}^{2}}$ and $\varsigma_{1}=1 / \sqrt{t_{R}^{2}+\left(1+\beta^{2}\right) t_{X}^{2}}$.

In the new basis $\left(A, Z_{L}, Z_{L}^{\prime}, Z_{R}, Z_{R}^{\prime}\right), A$ is decoupled, while $Z_{L}$ infinitesimally mixes with $\left(Z_{L}^{\prime}, Z_{R}, Z_{R}^{\prime}\right)$ where the relevant mixing angles are suppressed by $\left(u, u^{\prime}\right)^{2} /$ $\left(w, w_{R}, \Lambda_{R}\right)^{2} \ll 1$. Neglecting the mixing, $Z_{L}$ is a physical field and decoupled as the photon. We are left with diagonalizing the mass matrix of $\left(Z_{L}^{\prime}, Z_{R}, Z_{R}^{\prime}\right)$, which yields the eigenstates $\mathcal{Z}_{L}^{\prime}, \mathcal{Z}_{R}$, and $\mathcal{Z}_{R}^{\prime}$ and corresponding masses as

$$
\begin{gathered}
\mathcal{Z}_{L}^{\prime} \simeq Z_{L}^{\prime}, \quad \mathcal{Z}_{R} \simeq c_{\xi_{3}} Z_{R}-s_{\xi_{3}} Z_{R}^{\prime}, \quad \mathcal{Z}_{R}^{\prime} \simeq s_{\xi_{3}} Z_{R}+c_{\xi_{3}} Z_{R}^{\prime} \\
m_{\mathcal{Z}_{L}^{\prime}}^{2} \simeq \frac{g_{L}^{2}}{3} \frac{\left(1+\varsigma_{1}^{2} t_{R}^{2} t_{X}^{2} \beta^{2}\right)^{2} t_{W}^{2} w^{2}}{\varsigma_{1}^{2} t_{R}^{2} t_{X}^{2}}
\end{gathered}
$$

$$
m_{\mathcal{Z}_{R}}^{2} \simeq \frac{g_{L}^{2}}{3} \frac{3 w_{R}^{2}\left[t_{R}^{2}+t_{X}^{2}\left(1+\beta^{2}\right)\right]^{2}+w^{2}\left[\sqrt{3} t_{R}^{2}+(\sqrt{3}+\beta) t_{X}^{2}\right]^{2}}{\varsigma_{1}^{-2}\left[4+(\sqrt{3}+\beta)^{2}\left(t_{X}^{2} / t_{R}^{2}\right)\right]},
$$

$$
m_{\mathcal{Z}_{\mathcal{R}}^{\prime}}^{2} \simeq \frac{g_{L}^{2}}{3}\left[4 t_{R}^{2}+t_{X}^{2}(\sqrt{3}+\beta)^{2}\right] \Lambda_{R}^{2}
$$

provided that $\Lambda_{R} \gg w, w_{R}$, where the $Z_{R}-Z_{R}^{\prime}$ mixing angle is finite,

$$
t_{2 \xi_{3}}=\frac{2 t_{R}\left[\sqrt{3} t_{R}^{2}+\beta(3+\sqrt{3} \beta) t_{X}^{2}\right] \sqrt{t_{R}^{2}+t_{X}^{2}\left(1+\beta^{2}\right)}}{2 t_{R}^{4}+t_{R}^{2} t_{X}^{2}(\sqrt{3}-\beta)^{2}-\beta^{2}(\sqrt{3}+\beta)^{2} t_{X}^{4}} .
$$

Analogously, we can diagonalize the mass matrix for the case $\Lambda_{R} \ll w, w_{R}$, where $Z_{R}$ is decoupled, while $Z_{L, R}^{\prime}$ finitely mix. For the case $\Lambda_{R} \sim w, w_{R}$, all the gauge bosons $Z_{L, R}^{\prime}$ and $Z_{R}$ finitely mix, which can be parametrized by the Euler angles. Note that $w$ and $w_{R}$ are always taken in the same order, since they simultaneously break $S U(3)_{L} \otimes S U(3)_{R} \rightarrow S U(2)_{L} \otimes$ $S U(2)_{R}$ and correspondingly reduce the left-right symmetry.

\section{Higgs masses}

Let us rewrite the scalar potential that includes the full scalar content. The full scalar potential takes the form, $V=V_{\phi}+V_{\chi}+V_{\sigma}+V_{\text {mix }}$, where

$$
\begin{aligned}
V_{\phi}= & \mu_{\phi}^{2} \operatorname{Tr}\left(\phi^{\dagger} \phi\right)+\lambda_{1}\left[\operatorname{Tr}\left(\phi^{\dagger} \phi\right)\right]^{2}+\lambda_{2} \operatorname{Tr}\left[\left(\phi^{\dagger} \phi\right)^{2}\right], \\
V_{\chi}= & \mu_{\chi}^{2}\left[\left(\chi_{L}^{\dagger} \chi_{L}+\chi_{R}^{\dagger} \chi_{R}\right]+\kappa_{1}\left[\left(\chi_{L}^{\dagger} \chi_{L}\right)^{2}+\left(\chi_{R}^{\dagger} \chi_{R}\right)^{2}\right]+\kappa_{2}\left(\chi_{L}^{\dagger} \chi_{L}\right)\left(\chi_{R}^{\dagger} \chi_{R}\right),\right. \\
V_{\sigma}= & \mu_{\sigma}^{2}\left[\operatorname{Tr} \sigma_{L}^{\dagger} \sigma_{L}+\operatorname{Tr} \sigma_{R}^{\dagger} \sigma_{R}\right]+\rho_{1}\left\{\left[\operatorname{Tr}\left(\sigma_{L}^{\dagger} \sigma_{L}\right)\right]^{2}+\left[\operatorname{Tr}\left(\sigma_{R}^{\dagger} \sigma_{R}\right)\right]^{2}\right\}+\rho_{2}\left\{\operatorname{Tr}\left[\left(\sigma_{L}^{\dagger} \sigma_{L}\right)^{2}\right]+\operatorname{Tr}\left[\left(\sigma_{R}^{\dagger} \sigma_{R}\right)^{2}\right]\right\}+\rho_{3} \operatorname{Tr}\left[\sigma_{L}^{\dagger} \sigma_{L}\right] \operatorname{Tr}\left[\sigma_{R}^{\dagger} \sigma_{R}\right], \\
V_{\text {mix }}= & \zeta_{1}\left[\chi_{L}^{\dagger} \chi_{L}+\chi_{R}^{\dagger} \chi_{R}\right] \operatorname{Tr}\left(\phi^{\dagger} \phi\right)+\zeta_{2}\left[\chi_{L}^{\dagger} \chi_{L} \operatorname{Tr}\left(\sigma_{L}^{\dagger} \sigma_{L}\right)+\chi_{R}^{\dagger} \chi_{R} \operatorname{Tr}\left(\sigma_{R}^{\dagger} \sigma_{R}\right)\right]+\zeta_{3}\left[\chi_{L}^{\dagger} \sigma_{L} \sigma_{L}^{\dagger} \chi_{L}+\chi_{R}^{\dagger} \sigma_{R} \sigma_{R}^{\dagger} \chi_{R}\right] \\
& +\zeta_{4}\left[\chi_{L}^{\dagger} \chi_{L} \operatorname{Tr}\left(\sigma_{R}^{\dagger} \sigma_{R}\right)+\chi_{R}^{\dagger} \chi_{R} \operatorname{Tr}\left(\sigma_{L}^{\dagger} \sigma_{L}\right)\right]+\zeta_{5}\left[\chi_{L}^{\dagger} \phi \phi^{\dagger} \chi_{L}+\chi_{R}^{\dagger} \phi^{\dagger} \phi \chi_{R}\right]+\zeta_{6}\left[\operatorname{Tr}\left(\sigma_{L}^{\dagger} \sigma_{L}\right)+\operatorname{Tr}\left(\sigma_{R}^{\dagger} \sigma_{R}\right)\right] \operatorname{Tr}\left(\phi^{\dagger} \phi\right) \\
& +\zeta_{7}\left[\operatorname{Tr}\left(\phi^{\dagger} \phi \sigma_{R} \sigma_{R}^{\dagger}\right)+\operatorname{Tr}\left(\phi \phi^{\dagger} \sigma_{L} \sigma_{L}^{\dagger}\right)\right]+\zeta_{8}\left[\epsilon^{i j k} \epsilon_{\alpha \beta \gamma} \chi_{L i} \phi_{j}^{\alpha} \phi_{k}^{\beta} \chi_{R}^{\dagger \gamma}+\text { H.c. }\right] \\
& +\zeta_{9}\left[\sigma_{L i j} \phi_{\alpha}^{\dagger i} \phi_{\beta}^{\dagger j} \sigma_{R}^{\dagger \alpha \beta}+\text { H.c. }\right]+\left[f_{1} \epsilon^{i j k} \epsilon_{\alpha \beta \gamma} \phi_{i}^{\alpha} \phi_{j}^{\beta} \phi_{k}^{\gamma}+f_{2} \chi_{L}^{\dagger} \phi \chi_{R}+\text { H.c. }\right] .
\end{aligned}
$$

Here, the interactions $\zeta_{8,9}$ and $f_{1,2}$ are abnormal and subdominant since they can be removed by a global symmetry $U(1)$ that nontrivially transforms any one of the fields.

Expanding the neutral scalar fields around their VEVs, we find minimization conditions, mass terms, and interactions. The mass terms are divided as $V_{\text {mass }}=V_{S}+V_{A}+V_{\text {charged }}$, where $V_{S}$ and $V_{A}$ include those of $C P$-even and $C P$-odd scalar fields, respectively, whereas $V_{\text {charged }}$ consists of those of the charged scalars. Considering $q+1$-charged scalars, four fields $\left(\phi_{23}^{ \pm(q+1)}\right.$, $\phi_{32}^{ \pm(q+1)}, \chi_{R}^{ \pm(q+1)}$, and $\left.\chi_{L}^{ \pm(q+1)}\right)$ mix via a $4 \times 4$ matrix, which by diagonalization provides two massless Goldstone bosons, $G_{Y_{L}}^{ \pm(q+1)}$ and $G_{Y_{R}}^{ \pm(q+1)}$, and two massive Higgs fields, $\mathcal{H}_{1}^{ \pm(q+1)}$ and $\mathcal{H}_{2}^{ \pm(q+1)}$. These states are related to the gauge states by

$$
\left(\phi_{23}^{ \pm(q+1)} \phi_{32}^{ \pm(q+1)} \chi_{R}^{ \pm(q+1)} \chi_{L}^{ \pm(q+1)}\right)^{T}=\mathcal{P}\left(G_{Y_{L}}^{ \pm(q+1)} G_{Y_{R}}^{ \pm(q+1)} \mathcal{H}_{1}^{ \pm(q+1)} \mathcal{H}_{2}^{ \pm(q+1)}\right)^{T},
$$


where

$$
\mathcal{P} \simeq\left(\begin{array}{cccc}
0 & -\frac{\sqrt{\left(u^{\prime 2}-w^{2}\right)^{2}+w^{2} w_{R}^{2}}}{\sqrt{\left(u^{\prime 2}-w^{2}\right)^{2}+\left(w^{2}+u^{\prime 2}\right) w_{R}^{2}}} & 0 & \frac{u^{\prime 2} w_{R}}{\sqrt{\left(u^{\prime 2}-w^{2}\right)^{2}+\left(w^{2}+u^{\prime 2}\right) w_{R}^{2}}} \\
\frac{w^{2}-u^{\prime 2}}{\sqrt{\left.u^{\prime 2}-w^{2}\right)^{2}+w^{2} w_{R}^{2}}} & \frac{u^{\prime} w w_{R}}{\sqrt{\left.\left.\left(\left(u^{\prime 2}-w^{2}\right)^{2}+w^{2} w_{R}^{2}\right)\right)\left(u^{\prime 2}-w^{2}\right)^{2}+\left(w^{2}+u^{\prime 2}\right) w_{R}^{2}\right)}} & 0 & \frac{w w_{R}}{\sqrt{\left(u^{\prime 2}-w^{2}\right)^{2}+\left(w^{2}+w u^{\prime 2}\right) w_{R}^{2}}} \\
\frac{w w_{R} c_{\xi_{4}}}{\sqrt{\left.u^{\prime 2}-w^{2}\right)^{2}+w^{2} w_{R}^{2}}} & \frac{u^{\prime} w_{R}\left(u^{\prime 2}-w^{2}\right) c_{\xi_{4}}}{\sqrt{\left.\left(\left(u^{\prime 2}-w^{2}\right)^{2}+w^{2} w_{R}^{2}\right)\right)\left(\left(u^{\prime 2}-w^{2}\right)^{2}+\left(w^{2}+u^{\prime 2}\right) w_{R}^{2}\right)}} & -s_{\xi_{4}} & -\frac{\left(w^{2}-u^{\prime 2}\right) c_{\xi_{4}}}{\sqrt{\left(u^{\prime 2}-w^{2}\right)^{2}+\left(w^{2}+w u^{\prime 2}\right) w_{R}^{2}}} \\
-\frac{w w_{R} s_{\xi_{4}}}{\sqrt{\left.u^{\prime 2}-w^{2}\right)^{2}+w^{2} w_{R}^{2}}} & -\frac{u^{\prime} w_{R}\left(u^{\prime 2}-w^{2}\right) s_{\xi_{4}}}{\sqrt{\left.\left(\left(u^{\prime 2}-w^{2}\right)^{2}+w^{2} w_{R}^{2}\right)\right)\left(\left(u^{\prime 2}-w^{2}\right)^{2}+\left(w^{2}+u^{\prime 2}\right) w_{R}^{2}\right)}} & -c_{\xi_{4}} & \frac{\left(w^{2}-u^{\prime 2}\right) s_{\xi_{4}}}{\sqrt{\left(u^{\prime 2}-w^{2}\right)^{2}+\left(w^{2}+w u^{\prime 2}\right) w_{R}^{2}}}
\end{array}\right),
$$

where the mixing angle $\xi_{4}$ is defined by

$$
\tan 2 \xi_{4}=t_{2 \xi_{4}} \simeq-\frac{2 \zeta_{9} u \sqrt{u^{4}+u^{\prime 4}+2 u^{\prime 2} \Lambda_{R}^{2}-2 u^{2}\left(u^{\prime 2}-\Lambda_{R}^{2}\right)}}{\zeta_{7} \Lambda_{R}^{2} u^{\prime}} .
$$

Concerning the singly charged scalars, the model contains two massless Goldstone bosons $\left(G_{W_{L}}^{ \pm}\right.$and $\left.G_{W_{R}}^{ \pm}\right)$, which are eaten by $W_{L}^{ \pm}$and $W_{R}^{ \pm}$, respectively, and two physical massive fields, $H_{1}^{ \pm}$and $H_{2}^{ \pm}$. They are related to the gauge states through $\left(\sigma_{12 R}^{ \pm}, \phi_{12}^{ \pm}, \phi_{21}^{ \pm}, \sigma_{12 L}^{ \pm}\right)^{T}=\mathcal{K}\left(G_{W_{L}}^{ \pm}, G_{W_{R}}^{ \pm}, H_{1}^{ \pm}, H_{2}^{ \pm}\right)^{T}$, where

$$
\mathcal{K} \simeq\left(\begin{array}{cccc}
0 & -\frac{\sqrt{2\left(u^{2}+u^{\prime 2}\right)} \Lambda_{R}}{\sqrt{\left(u^{2}-u^{2}\right)^{2}+2 \Lambda_{R}^{2}\left(u^{2}+u^{\prime 2}\right)}} & -\frac{\left(u^{\prime 2}-u^{2}\right) s_{\xi_{5}}}{\sqrt{\left(u^{\prime 2}-u^{2}\right)^{2}+2 \Lambda_{R}^{2}\left(u^{2}+u^{\prime 2}\right)}} & \frac{\left(u^{\prime 2}-u^{2}\right) c_{\xi_{5}}}{\sqrt{\left(u^{\prime 2}-u^{2}\right)^{2}+2 \Lambda_{R}^{2}\left(u^{2}+u^{\prime 2}\right)}} \\
\frac{u^{\prime}}{\sqrt{u^{2}+u^{\prime 2}}} & -\frac{u\left(u^{2}-u^{\prime 2}\right)}{\sqrt{u^{2}+u^{\prime 2}} \sqrt{\left(u^{\prime 2}-u^{2}\right)^{2}+2 \Lambda_{R}^{2}\left(u^{2}+u^{\prime 2}\right)}} & -\frac{\sqrt{2} u \Lambda_{R} s_{\xi_{5}}}{\sqrt{\left(u^{\prime 2}-u^{2}\right)^{2}+2 \Lambda_{R}^{2}\left(u^{2}+u^{\prime 2}\right)}} & \frac{\sqrt{2} u \Lambda_{R} c_{\xi_{5}}}{\sqrt{\left(u^{\prime 2}-u^{2}\right)^{2}+2 \Lambda_{R}^{2}\left(u^{2}+u^{\prime 2}\right)}} \\
-\frac{u}{\sqrt{u^{2}+u^{\prime 2}}} & \frac{u^{\prime}\left(u^{\prime 2}-u^{2}\right)}{\sqrt{u^{2}+u^{\prime 2}} \sqrt{\left(u^{\prime 2}-u^{2}\right)^{2}+2 \Lambda_{R}^{2}\left(u^{2}+u^{\prime 2}\right)}} & -\frac{\sqrt{2} u^{\prime} \Lambda_{R} s_{\xi_{5}}}{\sqrt{\left(u^{\prime 2}-u^{2}\right)^{2}+2 \Lambda_{R}^{2}\left(u^{2}+u^{\prime 2}\right)}} & \frac{\sqrt{2} u^{\prime} \Lambda_{R} c_{\xi_{5}}}{\sqrt{\left(u^{\prime 2}-u^{2}\right)^{2}+2 \Lambda_{R}^{2}\left(u^{2}+u^{\prime 2}\right)}} \\
0 & 0 & c_{\xi_{5}} & s_{\xi_{5}}
\end{array}\right),
$$

with the mixing angel $\xi_{5}$ defined by

$$
t_{2 \xi_{5}} \simeq-\frac{2 u^{\prime} w w_{L} w_{R} \sqrt{\left(w^{2}+w_{L}^{2}\right)\left(w^{2}+w_{R}^{2}\right)}\left(w_{L}^{2}+w_{R}^{2}+2\left(w^{2}-u^{\prime 2}\right)\right)}{\left(w_{L}^{2}-w_{R}^{2}\right)\left(u^{\prime 4} w^{2}+u^{\prime 2} w_{L}^{2} w_{R}^{2}-w^{2}\left(w^{2}+w_{L}^{2}\right)\left(w^{2}+w_{R}^{2}\right)\right)} .
$$

The model also contains two heavy doubly charged scalars $H_{1}^{ \pm \pm}$and $H_{2}^{ \pm \pm}$, defined by

$$
H_{1}^{ \pm \pm}=c_{\xi_{7}} \sigma_{22 R}^{ \pm \pm}-s_{\xi_{7}} \sigma_{22 L}^{ \pm \pm}, \quad H_{2}^{ \pm \pm}=s_{\xi_{7}} \sigma_{22 R}^{ \pm \pm}+c_{\xi_{7}} \sigma_{22 L}^{ \pm \pm}
$$

where $t_{2 \xi_{7}}=\frac{2 u^{\prime 2} \zeta_{9} \Lambda_{L} \Lambda_{R}}{\left(\Lambda_{L}^{2}-\Lambda_{R}^{2}\right)\left(-u^{2} \zeta_{9}+2 \Lambda_{L} \Lambda_{R}\right)}$. Because of the limit $\Lambda_{R} \gg \Lambda_{L}$, the mixing angle $\xi_{7} \simeq 0$; hence, $\sigma_{22 L}$ and $\sigma_{22 R}$ are physical states by themselves.

For the neutral scalars, they split into two parts: $C P$ odd and $C P$ even. The model contains only a light $C P$-even neutral scalar that is identified as the standard model Higgs boson, while the other $C P$-even states achieve large masses at the new physical scale. Additionally, the $C P$-odd part contains four massless Goldstone bosons-which are correspondingly eaten by the four massive gauge bosons $Z, Z_{R}, Z_{L}^{\prime}$, and $Z_{R}^{\prime}$-and three heavy scalar states.

\section{FCNC}

As mentioned, the tree-level FCNCs arise due to the discrimination of quark generations; i.e., the third generations of left- and right-handed quarks $Q_{3 L, R}$ transform differently from the first two $Q_{\alpha L, R}$ under $S U(3)_{L, R} \otimes$ $U(1)_{X}$ gauge symmetry, respectively. Hence, the neutral currents will change ordinary quark flavors that nonuniversally couple to $T_{8 L, R}$, since $X$ is related to $T_{8 L, R}$ by the electric charge operator and $Q$ and $T_{3 L, R}$ conserve every flavor.

Indeed, with the aid of $X=Q-\left(T_{3 L}+T_{3 R}\right)-$ $\beta\left(T_{8 L}+T_{8 R}\right)$, the neutral currents of quarks take the form

$$
\begin{aligned}
\mathcal{L}_{\mathrm{NC}}= & -\bar{Q}_{L, R} \gamma_{\mu}\left[g_{L, R}\left(T_{3 L, R} A_{3 L, R}^{\mu}+T_{8 L, R} A_{8 L, R}^{\mu}\right)\right. \\
& \left.+g_{X}\left(Q-T_{3 L, R}-\beta T_{8 L, R}\right) B^{\mu}\right] Q_{L, R},
\end{aligned}
$$

where $Q_{L, R}$ are summed over all the quark multiplets. All the terms coupled to $T_{3 L, R}$ and $Q$ do not change flavor because $u_{L, R}$ and $d_{L, R}$ are identical under such charges. Hence, the FCNCs exist only for the terms that couple to $T_{8 L, R}$, 
$\mathcal{L}_{\mathrm{NC}} \supset-\sum_{a=1}^{3} \bar{Q}_{a L, R} \gamma_{\mu} T_{8 L, R} Q_{a L, R}\left(g_{L, R} A_{8 L, R}^{\mu}-\beta g_{X} B^{\mu}\right)$.

In the basis $\left(Z_{L}^{\prime}, Z_{R}, Z_{R}^{\prime}\right)$, the Lagrangian (50) is rewritten as

$$
\begin{aligned}
\mathcal{L}_{\mathrm{NC}} \supset & -\bar{q}_{L}^{\prime} \gamma_{\mu}\left(V_{q L}^{\dagger} T_{8 q} V_{q L}\right) q_{L}^{\prime}\left[g_{1} Z_{L}^{\prime \mu}+g_{2} Z_{R}^{\mu}+g_{3} Z_{R}^{\prime \mu}\right] \\
& -\bar{q}_{R}^{\prime} \gamma_{\mu}\left(V_{q L}^{\dagger} T_{8 q} V_{q R}\right) q_{R}^{\prime} g_{4} Z_{R}^{\prime \mu},
\end{aligned}
$$

where we denote $q^{\prime}$ as either $u^{\prime}$ or $d^{\prime}, T_{8 q}=\frac{1}{2 \sqrt{3}} \operatorname{diag}(1,1,-1)$, $g_{1}=g_{L} t_{R} t_{X} \cot \theta_{W} \varsigma_{1}, \quad g_{2}=g_{L} \beta t_{R} t_{X}^{2} \varsigma \varsigma_{1}, \quad g_{3}=-g_{L} t_{X}^{2} \beta^{2} \varsigma$, and $g_{4}=-\frac{g_{L}}{\varsigma}$.

Taking, for instance, the limit $\Lambda_{R}>w, w_{R}$ and changing to the mass basis, we obtain

$$
\begin{aligned}
\mathcal{L}_{\mathrm{NC}} \supset & -\bar{q}_{L}^{\prime} \gamma_{\mu}\left(V_{q L}^{\dagger} T_{8 q} V_{q L}\right) q_{L}^{\prime}\left[g_{1} Z_{L}^{\prime \mu}+\left(g_{2} c_{\xi_{3}}-g_{3} s_{\xi_{3}}\right) \mathcal{Z}_{R}^{\mu}\right. \\
& \left.+\left(g_{2} s_{\xi_{3}}+g_{3} c_{\xi_{3}}\right) \mathcal{Z}_{R}^{\prime \mu}\right] \\
& -\bar{q}_{R}^{\prime} \gamma_{\mu}\left(V_{q L}^{\dagger} T_{8 q} V_{q R}\right) q_{R}^{\prime} g_{4}\left(-s_{\xi_{3}} \mathcal{Z}_{R}^{\mu}+c_{\xi_{3}} \mathcal{Z}_{R}^{\prime \mu}\right) .
\end{aligned}
$$

It is noted that since $g_{R} A_{8 R}-\beta g_{X} B \sim Z_{R}^{\prime}$ and due to the large mixing $Z_{R}^{\prime}-Z_{R}$, both $\mathcal{Z}_{R}^{\prime}$ and $\mathcal{Z}_{R}$ contribute to the right current, whereas the left current composes $Z_{L}^{\prime}$ and these fields $\left(\mathcal{Z}_{R}\right.$ and $\left.\mathcal{Z}_{R}\right)$, as $g_{L} A_{8 L}-\beta g_{X} B$ is not orthogonal to $Z_{R}^{\prime}$. Consequently, the three fields $Z_{L}^{\prime}, \mathcal{Z}_{R}$, and $\mathcal{Z}_{R}^{\prime}$ dominantly couple to the tree-level FCNCs,

$$
\begin{aligned}
\mathcal{L}_{\mathrm{FCNC}}= & \frac{1}{\sqrt{3}} \bar{q}^{\prime}{ }_{i L} \gamma_{\mu} q_{j L}^{\prime}\left(V_{q L}^{*}\right)_{3 i}\left(V_{q L}\right)_{3 j}\left[g_{1} Z_{L}^{\mu}\right. \\
& \left.+\left(g_{2} c_{\xi_{3}}-g_{3} s_{\xi_{3}}\right) \mathcal{Z}_{R}^{\mu}+\left(g_{2} s_{\xi_{3}}+g_{3} c_{\xi_{3}}\right) \mathcal{Z}_{R}^{\prime \mu}\right] \\
& +\frac{1}{\sqrt{3}} \bar{q}^{\prime}{ }_{i L} \gamma_{\mu} q_{j L}^{\prime}\left(V_{q R}^{*}\right)_{3 i}\left(V_{q R}\right)_{3 j} g_{4}\left(-s_{\xi_{3}} \mathcal{Z}_{R}^{\mu}+c_{\xi_{3}} \mathcal{Z}_{R}^{\prime \mu}\right),
\end{aligned}
$$

for $i \neq j$. The new observation is that $Z_{R}$ changes flavor due to the large mixing with $Z_{R}^{\prime}$, in contrast to the minimal leftright symmetric model.

Integrating the heavy gauge bosons $\mathcal{Z}_{R}, \mathcal{Z}_{R}^{\prime}$, and $Z_{L}^{\prime}$ out, we determine the effective Lagrangian that describes the meson mixings,

$$
\mathcal{L}_{\mathrm{FCNC}}^{\mathrm{eff}}=-\Upsilon_{L}^{i j}\left({\overline{q^{\prime}}}_{i L} \gamma_{\mu} q_{j L}^{\prime}\right)^{2}-\Upsilon_{R}^{i j}\left(\bar{q}_{i R}^{\prime} \gamma_{\mu} q_{j R}^{\prime}\right)^{2},
$$

where

$$
\begin{aligned}
\Upsilon_{L}^{i j}= & \frac{1}{3}\left[\left(V_{q L}^{*}\right)^{3 i}\left(V_{q L}\right)^{3 j}\right]^{2}\left[\frac{g_{1}^{2}}{m_{Z_{L}^{\prime}}^{2}}+\frac{\left(g_{2} c_{\xi_{3}}-g_{3} s_{\xi_{3}}\right)^{2}}{m_{\mathcal{Z}_{R}}^{2}}\right. \\
+ & \left.\frac{\left(g_{2} s_{\xi_{3}}+g_{3} c_{\xi_{3}}\right)^{2}}{m_{\mathcal{Z}_{R}^{\prime}}^{2}}\right], \\
& \Upsilon_{R}^{i j}=\frac{1}{3}\left[\left(V_{q R}^{*}\right)^{3 i}\left(V_{q R}\right)^{3 j}\right]^{2}\left[\frac{g_{4}^{2} s_{\xi_{3}}^{2}}{m_{\mathcal{Z}_{R}}^{2}}+\frac{g_{4}^{2} c_{\xi_{3}}^{2}}{m_{\mathcal{Z}_{R}^{\prime}}^{2}}\right] .
\end{aligned}
$$

Generally, the fields $Z_{L}, Z_{R}$, and $Z_{R}^{\prime}$ mix via a $3 \times 3$ mass matrix, as given in Appendix. In this case, the mass eigenstates, $\mathcal{V} \equiv\left(\mathcal{Z}_{1}, \mathcal{Z}_{2}, \mathcal{Z}_{3}\right)$, are related to $V \equiv$ $\left(Z_{L}^{\prime}, Z_{R}, Z_{R}^{\prime}\right)$ by $V=U^{Z} \mathcal{V}$. Therefore, the couplings given in Eq. (56) are generalized by

$$
\begin{aligned}
\Upsilon_{L}^{i j}= & \frac{1}{3}\left[\left(V_{q L}^{*}\right)^{3 i}\left(V_{q L}\right)^{3 j}\right]^{2}\left[\frac{\left(g_{1} U_{11}^{Z}+g_{2} U_{21}^{Z}+g_{3} U_{31}^{Z}\right)^{2}}{m_{\mathcal{Z}_{1}}^{2}}\right. \\
& +\frac{\left(g_{1} U_{12}^{Z}+g_{2} U_{22}^{Z}+g_{3} U_{32}^{Z}\right)^{2}}{m_{\mathcal{Z}_{2}}^{2}} \\
& \left.+\frac{\left(g_{1} U_{13}^{Z}+g_{2} U_{23}^{Z}+g_{3} U_{33}^{Z}\right)^{2}}{m_{\mathcal{Z}_{3}}^{2}}\right],
\end{aligned}
$$

$\Upsilon_{R}^{\prime i j}=\frac{1}{3}\left[\left(V_{q R}^{*}\right)^{3 i}\left(V_{q R}\right)^{3 j}\right]^{2}\left[\frac{\left(g_{4} U_{31}^{Z}\right)^{2}}{m_{\mathcal{Z}_{1}}^{2}}+\frac{\left(g_{4} U_{32}^{Z}\right)^{2}}{m_{\mathcal{Z}_{2}}^{2}}+\frac{\left(g_{4} U_{33}^{Z}\right)^{2}}{m_{\mathcal{Z}_{3}}^{2}}\right]$.

This effective Lagrangian contributes to mass splittings $\Delta m_{M}$ between neutral mesons $M^{0}-\bar{M}^{0}$, where $M$ denotes $B_{d, s}$ or $K$. With the help of the mass matrix elements in Ref. [13], the mass differences computed from (57) and (58) are

$$
\begin{aligned}
\Delta m_{K} & =\frac{2}{3} \Re\left\{\Upsilon_{L}^{\prime 12}+\Upsilon_{R}^{\prime 12}\right\} m_{K} f_{K}^{2}, \\
\Delta m_{B_{d}} & =\frac{2}{3} \Re\left\{\Upsilon_{L}^{\prime 13}+\Upsilon_{R}^{\prime 13}\right\} m_{B_{d}} f_{B_{d}}^{2}, \\
\Delta m_{B_{s}} & =\frac{2}{3} \Re\left\{\Upsilon_{L}^{\prime 23}+\Upsilon_{R}^{\prime 23}\right\} m_{B_{s}} f_{B_{s}}^{2} .
\end{aligned}
$$

The total mass differences can be decomposed as

$$
\left(\Delta m_{M}\right)_{\mathrm{tot}}=\left(\Delta m_{M}\right)_{\mathrm{SM}}+\Delta m_{M},
$$

where the first term comes from the standard model contribution given in Ref. [14] and the second term is the new physics contribution as derived in (59)-(61). These predictions are compared to the experimental values [14]. Here, for the neutral kaon mixing, we assume that the theory predicts the mass difference within $30 \%$ since the potential longrange uncertainties are large. In contrast, the intrinsic theoretical uncertainties for $B_{s, d}$ mass differences are small, assumed to be within $5 \%$. In other words, the meson mass differences obey

$$
\begin{gathered}
0.37044 \times 10^{-2} / p s<\left(\Delta m_{K}\right)_{\mathrm{tot}}<0.68796 \times 10^{-2} / p s \\
0.480225 / p s<\left(\Delta m_{B_{d}}\right)_{\mathrm{tot}}<0.530775 / p s, \\
16.8692 / p s<\left(\Delta m_{B_{s}}\right)_{\mathrm{tot}}<18.6449 / p s
\end{gathered}
$$

For a numerical investigation, we take $w=w_{R}, g_{L}=g_{R}$ (i.e., $t_{R}=1$ ), $V_{u L}=V_{u R}=I$, and $\Lambda_{R}$ and $w$ are beyond the 

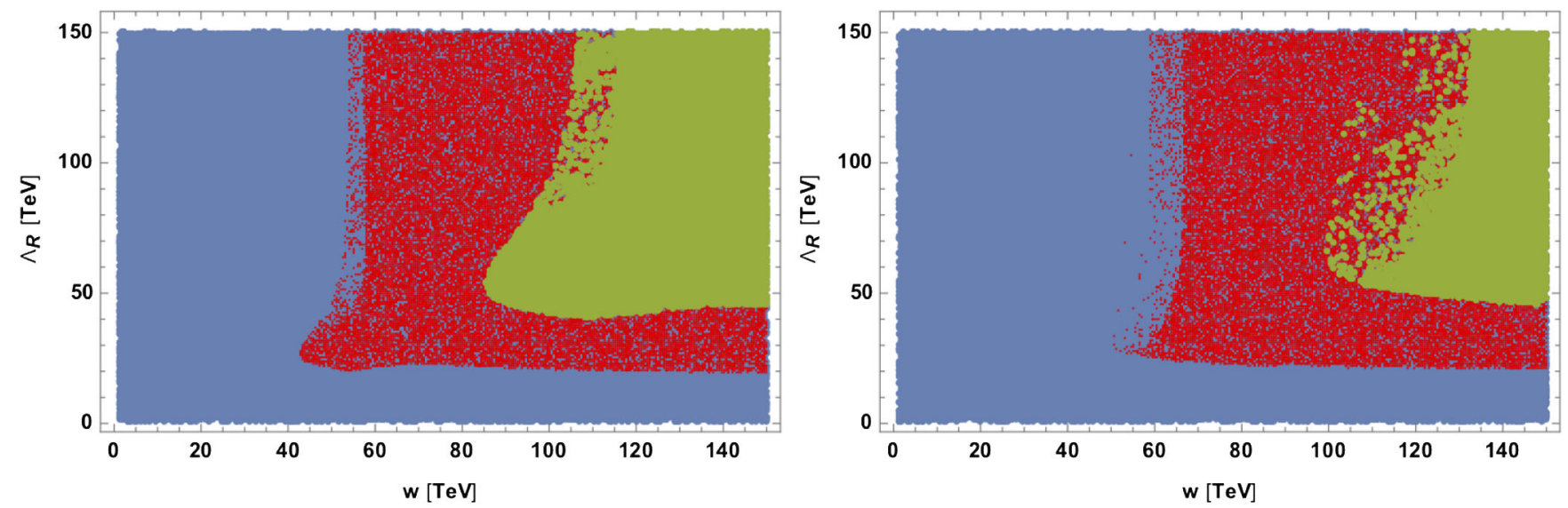

FIG. 1. Contours of $\Delta m_{K}, \Delta m_{B_{s}}$, and $\Delta m_{B_{d}}$ as a function of $\left(w, \Lambda_{R}\right)$ according to $\beta=-\frac{1}{\sqrt{3}}$ (left panel) and $\beta=\frac{1}{\sqrt{3}}$ (right panel).

weak scale and free to float. We have $V_{d L}=V_{d R}=V_{\mathrm{CKM}}$ (i.e., the misalignment in $V_{\mathrm{CKM}}$ tight to the down-quark sector), where the left and right values equal due to the leftright symmetry. With the input parameters $V_{\mathrm{CKM}}, m_{K, B_{s, d}}$, and $f_{K, B_{s, d}}$ given in Ref. [15] and the new neutral gauge boson masses derived by numerical diagonalization of the $M_{3 \times 3}$ matrix in Appendix, we make contours of the mass differences, $\Delta m_{K}$ and $\Delta m_{B_{d, s}}$ in the $w-\Lambda_{R}$ plane as in Fig. 1. The viable regime (gray) for the kaon mass difference is almost the whole frame. The red and olive regimes are viable for the mass differences $\Delta m_{B_{s}}$ and $\Delta m_{B_{d}}$, respectively. Combining all the bounds, we obtain $w>$ $85 \mathrm{TeV}$ and $\Lambda_{R}>54 \mathrm{TeV}$ for the model with $\beta=-\frac{1}{\sqrt{3}}$, whereas $w>99 \mathrm{TeV}$ and $\Lambda_{R}>66 \mathrm{TeV}$ for the model with $\beta=\frac{1}{\sqrt{3}}$. Here, the $\beta$ values chosen correspond to the dark matter versions [9].

\section{CHARGED LFV}

One of the strongest bounds on the charged LFV is the decay $\mu \rightarrow e \gamma$. Hence, in this work, we study that channel in detail and discuss other charged LFV processes which are potentially troublesome.

\section{A. $\mu \rightarrow e \gamma$ decay rate}

We are going to derive an expression for the branching decay ratio of $\mu \rightarrow e \gamma$ in the flipped trinification, based upon $S U(3)_{C} \otimes S U(3)_{L} \otimes S U(3)_{R} \otimes U(1)_{X}$ gauge symmetry, completed by a left-right symmetry of $S U(3)_{L}$ and $S U(3)_{R}$ interchange. Similarly to the standard model, the decay $\mu \rightarrow e \gamma$ in the present model cannot occur at tree level, but happens through one-loop diagrams, which are contributed by new Higgs scalars, new gauge bosons, and new leptons.

Suppose that the gauge states and the mass eigenstates of the new $(N)$ and ordinary charged $(l)$ leptons are related as

$$
\begin{aligned}
& N_{a L}=\left(U_{L}^{N}\right)_{a k} N_{k L}^{\prime}, \quad N_{a R}=\left(U_{R}^{N}\right)_{a k} N_{k R}^{\prime}, \\
& e_{a L}=\left(U_{L}^{l}\right)_{a k} e_{k L}^{\prime}, \quad e_{a R}=\left(U_{R}^{l}\right)_{a k} e_{k R}^{\prime},
\end{aligned}
$$

where $U_{L, R}^{N}$ and $U_{L, R}^{l}$ are basis-changing (mixing) matrices and unitary. If the left-right symmetry is not imposed, i.e., $w_{L}=0$ as in the previous study [9], $U_{L, R}^{N}$ and $U_{L, R}^{l}$ are not independent because the mass matrices of $N$ and $l$ are solely generated by the same Yukawa coupling $y_{a b}$ [cf. Eqs. (13) and (14)]. It is easily realized that the oneloop diagrams with the mediation of charged gauge $Y^{ \pm(q+1)}$ or Higgs $\mathcal{H}^{ \pm(q+1)}$ bosons that couple to $l$ and $N$ do not contribute to the decay $\mu \rightarrow e \gamma$, since the new leptons do not mix in the basis of charged lepton eigenstates due to the mentioned $m_{l}, m_{N} \sim y$. Alternatively, when the left-right symmetry is included, the mass matrices of $l$ and $N$ generally differ due to the $z_{a b}$ coupling contribution, in which one should note that $0 \neq w_{L} \ll w, w_{R} \sim M$ recognize a left-right asymmetry at the low energy. In this case, the new fields $Y^{ \pm(q+1)} / \mathcal{H}^{ \pm(q+1)}$ and $N$ significantly contribute. That said, the two cases must be taken into account when we parametrize the mixing matrices for numerical investigation, in the following section.

The neutrino mixing matrix is denoted as $U^{\nu}$, which is a $6 \times 6$ unitary matrix, relating the gauge state $X_{L} \equiv$ $\left(\nu_{L},\left(\nu_{R}\right)^{c}\right)^{T}$ to the mass eigenstate $X_{L}^{\prime}$, such as $X_{L}=U^{\nu} X_{L}^{\prime}$. We write $U^{\nu}$ in terms of

$$
U^{\nu}=\left(\begin{array}{cc}
U_{L} & U_{A} \\
U_{B} & U_{R}
\end{array}\right)=\left(\begin{array}{c}
U_{L}^{\nu} \\
U_{R}^{\nu}
\end{array}\right)
$$

Hence, the Yukawa coupling $x$ can be easily written in terms of diagonal light (called $m_{L}$ ) and heavy (called $m_{R}$ ) mass matrices and the mixing matrices $U_{L, R, A, B}$,

$$
x=-\frac{\left(U_{L}^{*} m_{L} U_{L}^{\dagger}+U_{A}^{*} m_{R} U_{A}^{\dagger}\right)}{\sqrt{2} \Lambda_{L}}=-\frac{\left(U_{R}^{*} m_{R} U_{R}^{\dagger}+U_{B}^{*} m_{L} U_{B}^{\dagger}\right)}{\sqrt{2} \Lambda_{R}} .
$$


TABLE I. Vertices that contribute to the decay rates $\ell \rightarrow \ell^{\prime} \gamma$.

\begin{tabular}{ll}
\hline \hline Vertex & \multicolumn{1}{c}{ Coupling } \\
\hline $\bar{e}_{L}^{\prime} X_{L}^{\prime c} H_{i}^{-}$ & $Y_{H_{i}^{-}}^{L}=-i\left(U_{L}^{l}\right)^{\dagger}(x y)\left(U^{\nu}\right)^{*} c_{\xi_{5}}\left(s_{\xi_{5}}\right)$ \\
$\bar{e}_{R}^{\prime} X_{L}^{\prime} H_{i}^{-}$ & $Y_{H_{i}^{-}}^{R}=-i\left(U_{R}^{l}\right)^{\dagger}(y x) U^{\nu} s_{\xi_{5}}\left(c_{\xi_{5}}\right)$ \\
$\bar{e}_{L}^{\prime} N_{R}^{\prime} \mathcal{H}_{i}^{-(q+1)}$ & $Y_{\mathcal{H}_{i}^{-(q+1)}}^{L}=-i\left(U_{L}^{l}\right)^{\dagger} y U_{R}^{N} c_{\xi_{4}}\left(s_{\xi_{4}}\right)$ \\
$\bar{e}_{R}^{\prime} N_{L}^{\prime} \mathcal{H}_{i}^{-(q+1)}$ & $Y_{\mathcal{H}_{i}^{-(q+1)}}^{R}=-i\left(U_{R}^{l}\right)^{\dagger} y U_{L}^{N} s_{\xi_{4}}\left(c_{\xi_{4}}\right)$ \\
$\left(\bar{e}_{L}^{\prime} \gamma^{\mu} \nu_{L}^{\prime}\right) W_{i \mu}^{-}$ & $\frac{-i g_{L}}{\sqrt{2}} U_{L}^{W_{i \mu}^{-}}=\frac{-i g_{L}}{\sqrt{2}}\left(U_{L}^{l \dagger} U_{L}^{\nu}\right) c_{\xi}\left(s_{\xi}\right)$ \\
$\left(\bar{e}_{R}^{\prime} \gamma^{\mu} \nu_{R}^{\prime}\right) W_{i \mu}^{-}$ & $\frac{-i g_{R}}{\sqrt{2}} U_{R}^{W_{i \mu}^{-}}=\frac{-i g_{R}}{\sqrt{2}}\left(U_{R}^{l \dagger} U_{R}^{\nu}\right) s_{\xi}\left(c_{\xi}\right)$ \\
$\left(\bar{e}_{L}^{\prime} \gamma^{\mu} N_{L}^{\prime}\right) Y_{i \mu}^{-(q+1)}$ & $\frac{-i g_{L}}{\sqrt{2}} U_{L}^{Y_{i \mu}^{-(q+1)}}=\frac{-i g_{L}}{\sqrt{2}}\left(U_{L}^{l \dagger} U_{L}^{N}\right) c_{\xi_{2}}\left(s_{\xi_{2}}\right)$ \\
$\left(\bar{e}_{R}^{\prime} \gamma^{\mu} N_{R}^{\prime}\right) Y_{i \mu}^{-(q+1)}$ & $\frac{-i g_{R}}{\sqrt{2}} U_{R}^{Y_{i \mu}^{-(q+1)}}=\frac{-i g_{R}}{\sqrt{2}}\left(U_{R}^{l \dagger} U_{R}^{N}\right) s_{\xi_{2}}\left(c_{\xi_{2}}\right)$ \\
$\bar{e}_{L}^{\prime} e_{L}^{\prime c} H_{i}^{--}$ & $Y_{H_{i}^{--}}^{L}=-i\left(U_{L}^{l}\right)^{\dagger} x\left(U_{L}^{l}\right)^{*} c_{\xi_{6}}\left(s_{\xi_{6}}\right)$ \\
$\bar{e}_{R}^{\prime} e_{R}^{\prime c} H_{i}^{--}$ & $Y_{H_{i}^{--}}^{R}=-i\left(U_{R}^{l}\right)^{\dagger} x\left(U_{R}^{l}\right)^{*} s_{\xi_{6}}\left(c_{\xi_{6}}\right)$ \\
\hline \hline &
\end{tabular}

Similarly, the Yukawa coupling $y$ can be expressed in terms of the diagonal matrices $m_{l}$ and $m_{N}$ that include respective charged and new lepton masses and the mixing matrices $U_{L, R}^{l, N}$,

$y=-\frac{\sqrt{2} U_{L}^{l} m_{l}\left(U_{R}^{l}\right)^{\dagger}}{u^{\prime}}=-\frac{\sqrt{2} U_{L}^{N} m_{N}\left(U_{R}^{N}\right)^{\dagger}}{w}-z \frac{w_{L} w_{R}}{\sqrt{2} M w}$.
To derive the decay rate $\mu \rightarrow e \gamma$ at one-loop approximation, we necessarily calculate the form factors of the relevant one-loop diagrams that contribute to the process. We list in Table I the vertices that are present in the current model and involved in the phenomenon of interest. In the table, we denote $i=1$ (2) according to either $c_{\xi}$ or $s_{\xi}$ out (in) the brackets, respectively. The previous works have calculated the form factors for the process $\mu \rightarrow e \gamma$ by taking into account the contributions of singly charged gauge bosons ( $W$ boson), doubly charged Higgs scalars [16-21], and singly charged Higgs scalars, where the last contribution was considered for the first time in Ref. [22]. In this paper, we present the results for the form factors of one-loop diagrams with the exchange of virtual general charged Higgs scalars and gauge bosons. To our best knowledge, this has not been done so far.

The effective Lagrangian derived from calculations of the form factors of one-loop diagrams for $\mu \rightarrow e \gamma$ with the participation of virtual scalars and gauge bosons in the considering model can be simply expressed as

$$
\mathcal{L}_{\text {eff }}=-4 \frac{e G_{F}}{\sqrt{2}} m_{\mu}\left(A_{R} \bar{e} \sigma_{\mu \nu} P_{R} \mu+A_{L} \bar{e} \sigma_{\mu \nu} P_{L} \mu\right) F^{\mu \nu}+\text { H.c. }
$$

Here, $A_{L, R}$ are the form factors,

$$
\begin{aligned}
A_{R}= & -\sum_{H^{Q}, k} \frac{1}{192 \sqrt{2} \pi^{2} G_{F} M_{H}^{2}}\left[\left(Y_{H}^{L}\right)_{\mu k}\left(Y_{H}^{L}\right)_{e k}^{*} \times F(Q)+\frac{m_{k}}{m_{\mu}}\left(Y_{H}^{R}\right)_{\mu k}\left(Y_{H}^{L}\right)_{e k}^{*} \times 3 \times F\left(r, s_{k}, Q\right)\right] \\
& +\sum_{A_{\mu}^{Q}, k} \frac{1}{32 \pi^{2}} \frac{M_{w}^{2}}{M_{A_{\mu}}^{2}}\left[\left(U_{A_{\mu}}^{L}\right)_{\mu k}\left(U_{A_{\mu}}^{L}\right)_{e k}^{*} G_{\gamma}^{Q}\left(\lambda_{k}\right)-\left(U_{A_{\mu}}^{R}\right)_{\mu k}\left(U_{A_{\mu}}^{L}\right)_{e k}^{*} \frac{m_{k}}{m_{\mu}} R_{\gamma}^{Q}\left(\lambda_{k}\right)\right], \\
A_{L}= & -\sum_{H^{Q}, k} \frac{1}{192 \sqrt{2} \pi^{2} G_{F} M_{H}^{2}}\left[\left(Y_{H}^{R}\right)_{\mu k}\left(Y_{H}^{R}\right)_{e k}^{*} \times F(Q)+\frac{m_{k}}{m_{\mu}}\left(Y_{H}^{L}\right)_{\mu k}\left(Y_{H}^{R}\right)_{e k}^{*} \times 3 \times F\left(r, s_{k}, Q\right)\right] \\
& +\sum_{A_{\mu}^{Q}, k} \frac{1}{32 \pi^{2}} \frac{M_{w}^{2}}{M_{A_{\mu}}^{2}} \frac{g_{R}^{2}}{g_{L}^{2}}\left[\left(U_{A_{\mu}}^{R}\right)_{\mu k}\left(U_{A_{\mu}}^{R}\right)_{e k}^{*} G_{\gamma}^{Q}(x)-\left(U_{A_{\mu}}^{L}\right)_{\mu k}\left(U_{A_{\mu}}^{R}\right)_{e k}^{*} \frac{m_{k}}{m_{\mu}} R_{\gamma}^{Q}\left(\lambda_{k}\right)\right],
\end{aligned}
$$

where $H^{Q}=H_{i}^{+}, H_{i}^{++}, \mathcal{H}_{i}^{+(q+1)}, A_{\mu}^{Q}=W_{i \mu}^{+}, Y_{i \mu}^{+(q+1)}(i=1,2)$, and $m_{k}$ are the masses of associated fermions that, along with either $H^{Q}$ or $A_{\mu}^{Q}$, form loops. The functions $F(Q), F\left(r, s_{k}, Q\right), G_{\gamma}^{Q}(x)$, and $R_{\gamma}^{Q}(x)$ appearing in Eqs. (71) and (72) are defined as

$$
\begin{gathered}
F(Q)=\frac{3}{4} Q-\frac{1}{2}, \\
F\left(r, s_{k}, Q\right)=Q-\frac{1}{2}-(Q-1) \times\left[\frac{4 s_{k}}{r}+\log \left(s_{k}\right)+\left(1-\frac{2 s_{k}}{r}\right) \sqrt{1+\frac{4 s_{k}}{r}} \log \left(\frac{\sqrt{r+4 s_{k}}+\sqrt{r}}{\sqrt{r+4 s_{k}}-\sqrt{r}}\right)\right], \\
G_{\gamma}^{Q}(x)=\frac{(9 Q+2) x^{2}-(12 Q-5) x+3 Q-1}{4(x-1)^{3}}-\frac{3}{2} \frac{x^{2}(Q x-Q+1)}{(x-1)^{4}} \log (x),
\end{gathered}
$$




$$
\begin{aligned}
R_{\gamma}^{Q}(x)= & -\frac{(2 Q-1) x^{2}+(2 Q-1) x-4(Q+1)}{2(x-1)^{2}} \\
& +\frac{3 x(Q x-Q-1)}{(x-1)^{3}} \log (x),
\end{aligned}
$$

where we have defined $\lambda_{k}=m_{k}^{2} / M_{A_{\mu}^{Q}}^{2}, s_{k}=m_{k}^{2} / M_{H^{Q}}^{2}$, $r=-q^{2} / M_{H^{Q}}^{2}$, and that $q=p_{2}-p_{1}$ is transferred momentum.

The branching ratio of $\mu \rightarrow e+\gamma$ decay is obtained as $[17,18]$

$$
\operatorname{Br}(\mu \rightarrow e+\gamma)=384 \pi^{2}\left(4 \pi \alpha_{\mathrm{em}}\right)\left(\left|A_{R}\right|^{2}+\left|A_{L}\right|^{2}\right),
$$

where $\alpha_{\mathrm{em}}=1 / 128$ is the fine-structure constant.

\section{B. Numerical analysis/discussion: $w_{L}=0$}

Before performing numerical calculations using the branching decay formula obtained in the previous section, let us estimate the magnitudes of relevant VEVs. Among the VEVs introduced, the smallest one could be $\Lambda_{L}$, which is at electron-volt scale responsible for the neutrino masses, much smaller than the weak scales $u$ and $u^{\prime}$ satisfying the constraint $u^{2}+u^{\prime 2}=(246 \mathrm{GeV})^{2}$. Hence, we safely neglect the contributions of $\Lambda_{L}$. The quark FCNC constraints imply $w, w_{R}, \Lambda_{R} \gtrsim \mathcal{O}(50-100) \mathrm{TeV}$, appropriate for the collision bounds [9], in which such VEVs break the flipped trinification to the standard model, significantly greater than the weak scales. Finally, $\Lambda_{R}$ can take a value, such that (i) $\Lambda_{R} \gg w, w_{R}$, (ii) $\Lambda_{R} \sim w, w_{R}$, or (iii) $\Lambda_{R} \ll w$, $w_{R}$, depending on the symmetry breaking scheme. The viable dark mater scenarios $[9,23]$ prefer cases $i$ and ii, which will be taken into account.

Because of the condition $\Lambda_{R}, w, w_{R} \gg u, u^{\prime} \gg w_{L}, \Lambda_{L}$, the masses of the gauge bosons relevant to the process are approximated as $m_{W_{1}}^{2} \simeq \frac{g^{2}}{4}\left(u^{2}+u^{\prime 2}\right), m_{W_{2}}^{2} \simeq \frac{g^{2}}{2} \Lambda_{R}^{2}, m_{Y_{1}}^{2} \simeq \frac{g^{2}}{4} w^{2}$, and $m_{Y_{2}}^{2} \simeq \frac{g^{2}}{4}\left(w^{2}+w_{R}^{2}\right)$, where we have used $g_{L}=g_{R}=g$. Note that $W_{1}$ has the mass identical to the standard model, while $m_{W_{2}}$ and $m_{Y_{1,2}}$ are large, at tera-electron-volt scale or higher. The masses of relevant new Higgs bosons $H^{ \pm}, H^{ \pm \pm}$, and $H^{ \pm(q+1)}$ depend on unknown parameters present in the scalar potential, which cannot be estimated precisely. However, their masses are all proportional to the new physics scales $\Lambda_{R}, w$, and $w_{R}$, which should be large enough to escape detection [15]. That said, it is reasonable to choose the new Higgs masses from hundreds of giga-electron-volts to a few tera-electron-volts. Particularly, in hierarchical cases, the largest masses can be chosen up to hundreds of teraelectron-volts.

Let us parametrize the Yukawa couplings and mixing matrices, involved in the branching ratio $\mu \rightarrow e \gamma$ in (77), in forms convenient for numerical investigation using the current data. Without lost of generality, we work in the basis of charged lepton mass eigenstates, i.e., $m_{l}=y u^{\prime} / \sqrt{2}$ is diagonal, implying $U_{L, R}^{l}=I$, in the same criteria used in the standard model. Besides, the new lepton masses are generated by the same Yukawa matrix $y$, with the relation between both kinds of masses given in (69), where $w_{L}=0$. Hence, the choice of $U_{L, R}^{l}$ leads to $U_{L, R}^{N}=I$. Without depending of basis, the ratio $m_{l}^{i} / m_{N}^{i}(i=1,2,3)$ is universal for any generation,

$$
\frac{m_{l}^{i}}{m_{N}^{i}}=\frac{u^{\prime}}{w}
$$

which implies $m_{N}^{i} \gtrsim 50 \mathrm{MeV}, 10 \mathrm{GeV}$, and $170 \mathrm{GeV}$ for $i=1,2,3$, respectively, given that $w / u^{\prime} \gtrsim 100$. We are interested in the two dark matter versions according to $\beta= \pm 1 / \sqrt{3}$, where we note that $N$ is a standard model singlet for $\beta=-1 / \sqrt{3}$, whereas it has an electric charge $q=-1$ for $\beta=1 / \sqrt{3}$. The former is always viable, similarly to the case of a light sterile neutrino. However, the latter should be ruled out due to the electroweak precision test, unless the new physics scale is unexpectedly raised, $w / u^{\prime} \gtrsim 10^{5}$, so that the lightest new lepton is heavy enough to suppress the dangerous processes, e.g., $Z \rightarrow N N$.

Note that at one-loop approximations the diagrams with virtual neutral Higgs scalars do not contribute to LFV processes, including $\mu \rightarrow e \gamma$ decay, because the interacting vertices of two leptons with such a neutral scalar do not change flavor (i.e., conserving flavor). The vertex couplings are governed by the magnitudes of diagonal elements of the Yukawa matrix $y$ as well as a mixing factor among neutral scalars. These vertices are also constrained by the current experiments through the channels of the standard model-like Higgs decay into two leptons $h \rightarrow \ell \bar{\ell}^{\prime}$. According to Ref. [15], $h \rightarrow \tau \bar{\tau}$ has been observed at a quite high precision, while $h \rightarrow \mu \bar{\mu}$ is likely observed, but at large uncertainty, and the branching decay $h \rightarrow e \bar{e}$ can only be set by an upper limit, $\operatorname{Br}(h \rightarrow e \bar{e})<1.9 \times 10^{-3}$. All these agree with the strengths of $h \ell \ell$ interactions, set by the corresponding lepton masses. Because of the mixing, $h$ can decay into light $N$ 's, but the rate is highly suppressed by $\left(u, u^{\prime}\right)^{2} /\left(w, w_{R}, \Lambda_{R}\right)^{2} \ll 1$. The light $N$ 's are undetectable due to weak interaction strengths. However, since no constraint has been placed on their masses, they can take any values consistent with the scenario of interest.

Our study is interested in a model in which the new physics scale is not too high, thus presenting rich physical phenomena at the current and future experiments. Let us fix the benchmark values for $u^{\prime}$ and $w$, based upon the relation (78) and the others. Close to the standard model, we choose $u^{\prime}$ near its maximum, $u^{\prime} \simeq 246 \mathrm{GeV}$. Another advantage of this choice leads to the smallness of $u$, thus having a significant reduction of $\Lambda_{R}$ satisfying the required condition for the seesaw mechanism, $u^{2} / \Lambda_{R} \sim \mathrm{eV}$. Choosing 
the lower bound for the heaviest new lepton to be 50,200, or $500 \mathrm{GeV}$, one has $w \geq 7,28$, or $70 \mathrm{TeV}$, respectively. In the seesaw mechanism, note that $U_{L}$ and $U_{R}$ diagonalize the mass matrix $M_{\nu}$ and $M_{R}$, respectively. Thus, $U_{L}$ and $U_{R}$ generally differ. The observed neutrino masses imply the sizes of $M_{L}$ and $M_{D}^{T} M_{R}^{-1} M_{D}$ in electron-volts. The smallness of $M_{L}$ is ensured by small $\Lambda_{L}$, say $\Lambda_{L} \sim 1 \mathrm{eV}$, while the magnitude of $M_{D}^{T} M_{R}^{-1} M_{D}$ depends on the correlation between $y, u$, and $M_{R}$. Since $y$ has been fixed before $\operatorname{diag}(y)=\left(3 \times 10^{-6}, 6 \times 10^{-4}, 10^{-2}\right)$, the lower bound set for the right-handed neutrino masses $M_{R}$ is 10 and $1000 \mathrm{TeV}$ according to $u=0.1$ and $1 \mathrm{GeV}$, respectively.

Summarizing all, the parmetrization for numerical investigation is now performed. First of all, $U_{L}$ coincides with the Pontecorvo-Maki-Nakagawa-Sakata matrix $U_{\text {PMNS }}$, determined with a high accuracy by the oscillation experiments, except for the Dirac $C P$-violation phase (where the Majorana $C P$-violation phases are neither determined nor contributing to the process). The $3 \times 3$ unitary matrix $U_{R}$ is parametrized in the same way as $U_{\text {PMNS }}$, but its angles and phase are freely chosen in the calculation. Last, $M_{R}$ can be calculated using the relation $M_{R}=U_{R}^{*} M_{R}^{\text {diag }} U_{R}^{\dagger}$, where $M_{R}^{\text {diag }}$ has a diagonal form of the heavy neutrino masses. The Yukawa matrix $x$ is derived from $M_{R}$ as $x=$ $-U_{R}^{*} M_{R}^{\text {diag }} U_{R}^{\dagger} /\left(\sqrt{2} \Lambda_{R}\right)$. In the following, we will present the results of numerical calculations for the case in which the involved parameters are chosen as $u=0.1 \mathrm{GeV}$, $w=10 \mathrm{TeV}, \quad w_{R}=20 \mathrm{TeV}, \operatorname{diag}\left(M_{R}^{\mathrm{diag}}\right)=(10,20,30) \mathrm{TeV}$, and $U_{R}\left(\theta_{12}^{\prime}, \theta_{13}^{\prime}, \theta_{23}^{\prime}, \delta^{\prime}\right)=U_{R}(\pi / 4, \pi / 4,0,0)$. Note that the choice $u=0.1 \mathrm{GeV}$ is in order to conserve the condition $u^{\prime} \simeq 246 \mathrm{GeV}$, the important implications of which have been discussed before. The other quantities such as $\Lambda_{L}, w_{L}$, and light neutrino masses are neglected due to the small effects for the process.

Although $Y_{i}$ and $\mathcal{H}_{i}$ are listed in Table I, it is realized that their vertices do not contribute to the $\mu \rightarrow e \gamma$ branching ratio at the one-loop level, as mentioned. The reason is similar to the case of vertices of neutral Higgs scalars, which conserve lepton flavors. Indeed, all of the matrices relevant to them, such as $U_{R, L}^{l}, U_{R, L}^{N}$, and $y$, are diagonal.

In Figs. 2-5, we respectively show the dependence of the branching ratio $\operatorname{Br}(\mu \rightarrow e \gamma)$ on the relevant parameters in this kind of model. To produce the results, we have separately considered the contributions to the decay rate corresponding to the exchanges of the virtual gauge boson and charged Higgs scalar. As has been introduced above and expressed in detail from Eq. (70)-(77), in the model under consideration, the $\mu \rightarrow e \gamma$ branching ratio depends complicatedly on many parameters, most of which are unknown. Moreover, the variation of a parameter might change the contribution of the involving channel to few orders, e.g., the mixing angles between the left and right sectors $\xi$; see the figures for details. Therefore, presenting individual contributions would provide more information

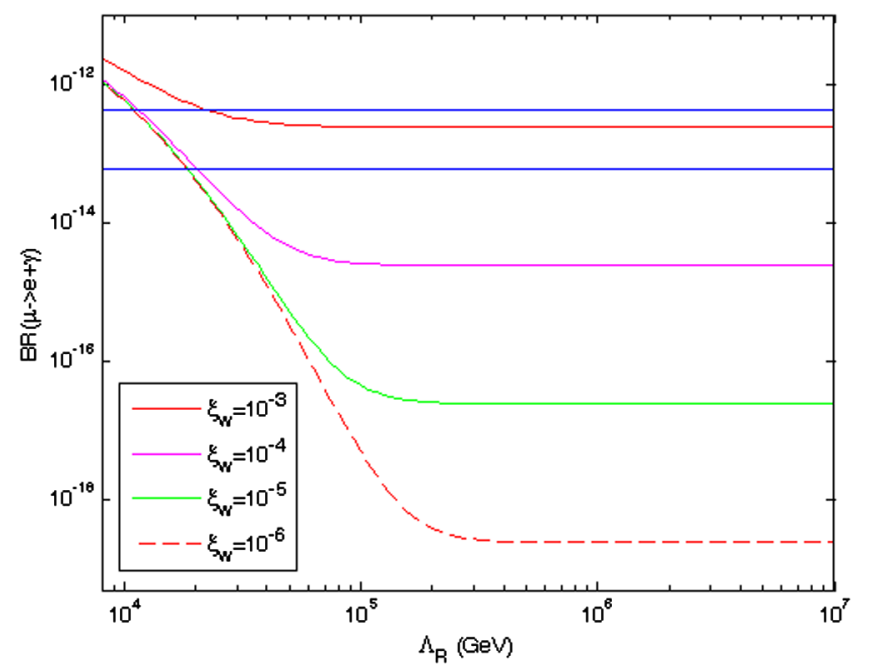

FIG. 2. The branching ratio $\operatorname{Br}(\mu \rightarrow e \gamma)$ governed by intermediate $W_{1,2}^{ \pm}$gauge bosons, which is given as a function of $\Lambda_{R}$ for the selected values of their mixing angle $\xi_{w}$. The upper and lower blue lines correspond to the MEG current bound and near-future sensitivity limit.

and better understanding about the phenomenon. We also suppose that heavy Higgs $H_{i}^{ \pm}$and $H_{i}^{ \pm \pm}(i=1,2)$ possess equivalent masses, commonly called $M_{H}$. Additionally, the mixing angles between $H_{1}^{ \pm}$and $H_{2}^{ \pm}$as well as between $H_{1}^{ \pm \pm}$and $H_{2}^{ \pm \pm}$are equally taken and denoted as $\xi_{H}$.

Figure 2 describes the dependence of $\mu \rightarrow e \gamma$ upon the diagrams that exchange virtual gauge bosons $W_{i}(i=1,2)$,

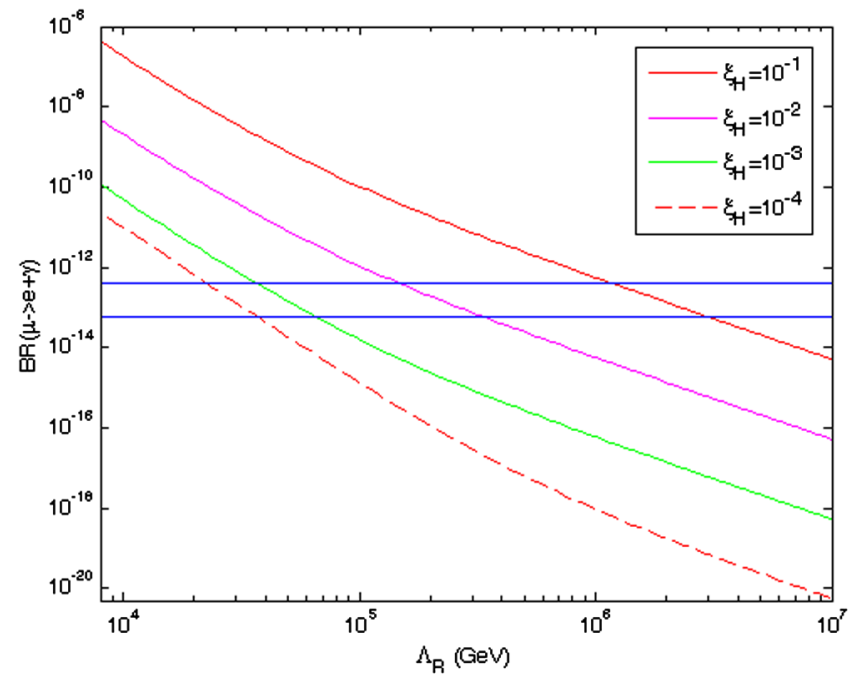

FIG. 3. The branching ratio $\operatorname{Br}(\mu \rightarrow e \gamma)$ governed by intermediate Higgs bosons $H_{1,2}^{ \pm}$and $H_{1,2}^{ \pm \pm}$, which is given as a function of $\Lambda_{R}$ for the selected values of the mixing angle $\xi_{H}$. Here, we have set $M_{H}=10 \mathrm{TeV}$ as a common mass for all $H_{1,2}^{ \pm}$and $H_{1,2}^{ \pm \pm}$ and supposed that the pairs $H_{1}^{ \pm}-H_{2}^{ \pm}$and $H_{1}^{ \pm \pm}-H_{2}^{ \pm \pm}$have the same mixing angle $\left(\xi_{H}\right)$. 

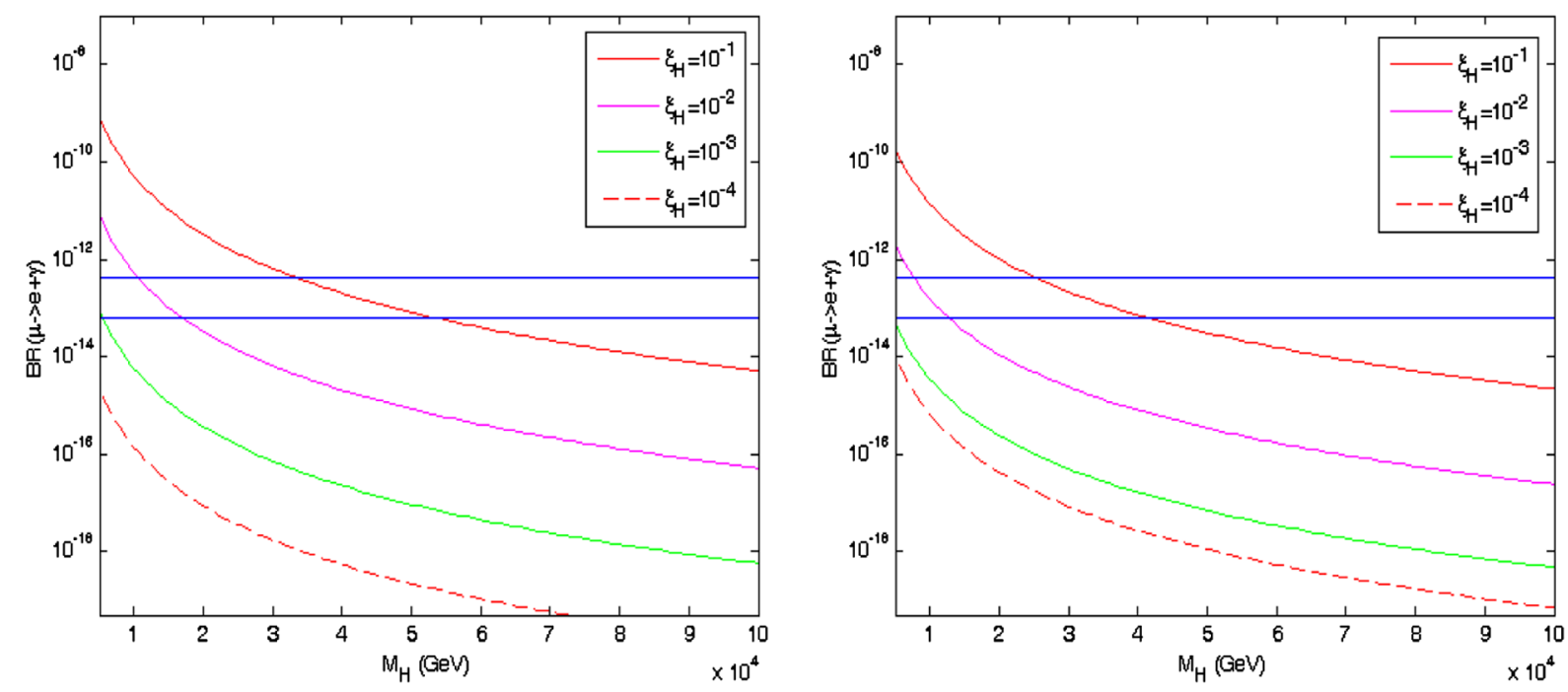

FIG. 4. The branching ratio $\operatorname{Br}(\mu \rightarrow e \gamma)$ governed by either virtual singly charged scalars (left panel) or virtual doubly charged scalars (right panel), all given as a function of the Higgs common mass $M_{H}$ (all the Higgs bosons presumably have the same mass) for different values of their mixing angle $\xi_{H}$ with fixed $\Lambda_{R}=100 \mathrm{TeV}$.

given as a function of $\Lambda_{R}$. The choice of $\operatorname{diag}\left(M_{R}^{\text {diag }}\right)=$ $(10,20,30) \mathrm{TeV}$ and $U_{L, R}$ has canceled out the dependence of $U_{\text {mix }}$ on $\Lambda_{R}$. Moreover, $W_{1}$ is the standard model $W$ boson of which the mass is fixed as $M_{W_{1}}=80 \mathrm{GeV}$. Therefore, the branching ratio lines shown in Fig. 2 are depicted as a function of the new boson mass $M_{W_{2}} \sim \Lambda_{R}$ and mixing angle $\xi_{w}$. For each value of $\xi_{w}$, the branching ratio goes down due to the dominant contribution of $W_{2}$ to a constant value, as increasing $\Lambda_{R}$. The constant line is preserved by a constant contribution of $W_{1}$. Using the $\mathrm{Mu}$ to E Gamma experiment (MEG) current bound on the

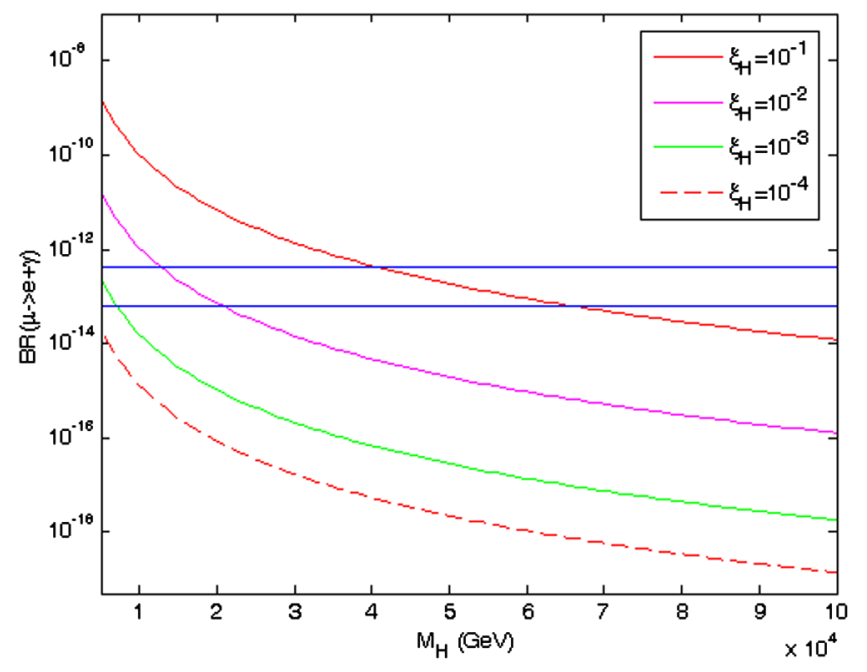

FIG. 5. Dependence of the branching ratio $\operatorname{Br}(\mu \rightarrow e \gamma)$ as governed by the exchanges of virtual singly and doubly charged scalars, given as a function of the Higgs common mass $M_{H}$ for different cases of the mixing angle $\xi_{H}$, where $\Lambda_{R}=100 \mathrm{TeV}$ is fixed. $\mu \rightarrow$ er decay, one roughly estimates the lower bound $\Lambda_{R} \geq$ 12 and $10 \mathrm{TeV}$ for $\xi_{w}=10^{-3}$ and $\xi_{w} \leq 10^{-4}$, respectively. The strong dependence of the branching ratio on the mixing angle $\xi_{w}$, which separates about two orders between two successive lines for the range of large $\Lambda_{R}$, suggests the domination of the interference terms in $A_{L, R}$ [cf. Eqs. (71) and (72)]. Indeed, the interference terms are proportional to $\frac{m_{k}}{m_{\mu}} \sin \xi_{w} \cos \xi_{w} \approx \frac{m_{k}}{m_{\mu}} \xi_{w}$. Thus, the branching ratio is proportional to $\frac{m_{k}^{2}}{m_{\mu}^{2}} \xi_{w}^{2} \sim \xi_{w}^{2}$, which is consistent with the observation from the figure, whereas the other terms are either proportional to $\cos \xi_{w}^{2} \simeq 1$ or suppressed by a factor $\xi_{w}^{2}$. It is figured out that the dominant interference terms are provided by the factor $\frac{m_{k}}{m_{\mu}} \sim 10^{5}$, for instance, for the case of heavy neutrino mass $m_{k} \sim 10^{4} \mathrm{GeV}$. Similarly, we have the same domination of the interference terms in Figs. 3-5.

The branching ratio in Fig. 3 is a monotonically decreasing function of $\Lambda_{R}$, which enters the decay rate through the interaction vertices, which have strengths depending on the elements of the Yukawa coupling matrix $x \sim 1 / \Lambda_{R}$. The behavior of the branching ratio is considered for different values of the mixing angle $\xi_{H}$. The figure implies that, consistent with the current MEG upper bound, $\operatorname{Br}(\mu \rightarrow e \gamma)<4.2 \times 10^{-13}$, the lower limits are $\Lambda_{R} \geq 1100,105,13,10.2 \mathrm{TeV}$ according to $\xi_{H}=10^{-1}$, $10^{-2}, 10^{-3}, 10^{-4}$, respectively. Besides the sensitivity of the future MEG are possible to probe $\mu \rightarrow e \gamma$ signal, provided that $\Lambda_{R} \leq 1300,120,15.5,13 \mathrm{TeV}$ for $\xi_{H}=10^{-1}, 10^{-2}$, $10^{-3}, 10^{-4}$, respectively.

In the next two figures, we demonstrate the dependence of the branching ratio as a single variable function of the new Higgs mass $M_{H}$, where $\Lambda_{R}$ is fixed as $100 \mathrm{TeV}$. As we see from Figs. 4 and 5, the smaller the mixing angle $\xi_{H}$ is, 
the smaller the lower bound is set for the heavy Higgs masses. If the contributions to the diagrams include only the virtual singly charged scalar (Fig. 4, left panel), the lower bound for the scalar masses reduces from $M_{H}=$ $53 \mathrm{TeV}$ at $\xi=10^{-1}$ down to $M_{H}<10 \mathrm{TeV}$ at $\xi=10^{-4}$. We get almost the same limits for the case with doubly charged scalar exchanges (Fig. 4, right panel).

\section{Numerical analysis/discussion: $w_{L} \neq 0$}

The flipped trinification discriminates from the minimal left-right symmetric model especially in the extended particle sectors, governed by the new gauge symmetry. Part of them produces the interesting quark FCNCs, as studied above. In this section, we argue that the presence of other part of them gives novel contributions to the charged LFV. It is stressed that such LFV processes, e.g., $\mu \rightarrow e \gamma$, can be altered in the case of nonvanishing $w_{L}$. Although $w_{L}$ is constrained to be much smaller than $M, \Lambda_{R}, w_{R}$, and $w$ as well as not modifying the results discussed in the previous section related to $H_{i}$ and $W_{i}$, the nonvanishing $w_{L}$ causes the mass matrices of ordinary charged and new leptons to be not simultaneously diagonalized. This provides the new sources of the LFV, which involve the $(q+1)$-charged Higgs and gauge bosons $\left(\mathcal{H}_{1,2}, Y_{1,2}\right)$ as well as the new leptons $(N)$ in the loops for $\mu \rightarrow e \gamma$, which is a new feature of the model.

In the basis of ordinary charged lepton mass eigenstates, the mentioned, new lepton mass matrix can be expressed as

$$
M_{N}=M_{N}^{0}+\Delta M_{N}
$$

where

$$
\begin{gathered}
M_{N}^{0}=-\frac{y_{a b}}{\sqrt{2}} w=\left(\begin{array}{ccc}
m_{N 1}^{0} & 0 & 0 \\
0 & m_{N 2}^{0} & 0 \\
0 & 0 & m_{N 3}^{0}
\end{array}\right), \\
\Delta M_{N}=-\frac{z_{a b}}{2 M} w_{L} w_{R}=\left(\begin{array}{ccc}
a_{1} & b_{1} & b_{2} \\
B_{1} & a_{2} & b_{3} \\
B_{2} & B_{3} & a_{3}
\end{array}\right) .
\end{gathered}
$$

Here, $\|y\| \sim\left\|M_{l}\right\| / u^{\prime} \sim 10^{-3}-10^{-2}$ is constrained by the ordinary charged lepton masses and small. The coupling matrix $z$ is generic and maybe sizable, but it generally obeys $\|z\| \sim 1 \ll\|y\|\left(w / w_{L}\right)$, provided that $w / w_{L} \gtrsim 10^{3}$. For instance, if $w=10 \mathrm{TeV}$, one takes $w_{L} \lesssim 10 \mathrm{GeV}$. This leads to $\left\|\Delta M_{N}\right\| \ll\left\|M_{N}^{0}\right\|$, which is also expected due to the contribution of the effective interactions. To find the mixing matrix, we pertubatively dialgonalize the squared mass matrix, $M M^{\dagger}$, while taking into account $\Delta M_{N}$ as a subdominant contribution compared to $M_{N}^{0}$. The final result is

$$
U \approx\left(\begin{array}{ccc}
1 & \frac{b_{1}^{*} m_{N 1}^{0}+B_{1} m_{N 2}^{0}}{\left(m_{N 2}^{0}\right)^{2}-\left(m_{N 1}^{0}\right)^{2}} & \frac{b_{2}^{*} m_{N 1}^{0}+B_{2} m_{N 3}^{0}}{\left(m_{N 3}^{0}\right)^{2}-\left(m_{1}^{0}\right)^{2}} \\
-\frac{b_{1} m_{N 1}^{0}+B_{1}^{*} m_{N 2}^{0}}{\left(m_{N 2}^{0}\right)^{2}-\left(m_{N 1}^{0}\right)^{2}} & 1 & \frac{b_{3}^{*} m_{N 2}^{0}+B_{3} m_{N 3}^{0}}{\left(m_{N 3}^{0}\right)^{2}-\left(m_{2}^{0}\right)^{2}} \\
-\frac{b_{2} m_{N 1}^{0}+B_{2}^{*} m_{N 3}^{0}}{\left(m_{N 3}^{0}\right)^{2}-\left(m_{1}\right)^{2}} & -\frac{b_{3} m_{N 2}^{0}+B_{3}^{*} m_{N 3}^{0}}{\left(m_{N 3}\right)^{2}-\left(m_{2}^{2}\right)^{2}} & 1
\end{array}\right) .
$$

For brevity, in numerical calculation, we assume $z_{a b}$ as a real symmetric matrix with $z_{12}=z_{13}=z_{23}=1$, which means that the new lepton mass matrix is invariant under the charge-conjugation and parity transformations. This choice leads to an approximation, $U_{L}^{E} \simeq U_{R}^{E} \simeq U$. We fix $M=100 \mathrm{TeV}, w=30 \mathrm{TeV}$, and $w_{L}$ appropriately ranging from an infinitesimal value to a few giga-electron-volts. All the remaining parameters take the same values as in the previous subsection.

In Fig. 6, we depict the dependence of the branching ratio $\operatorname{Br}(\mu \rightarrow e \gamma)$, contributed by the exchanges of virtual $Y_{1,2}^{ \pm(q+1)}$ gauge bosons, in terms of $w_{L}$ for several values of the mixing angle $\xi_{Y}$. With the set of the parameters used in the numerical calculation, the branching ratio of $\mu \rightarrow e \gamma$ is within the sensitivities of the current and near-future experiments. The upper bounds $w_{L}=0.35,3.53 \mathrm{GeV}$ are set for $\xi_{Y}=10^{-1}, 10^{-2}$, respectively. While the next MEG upgrade might probe the decay signal if $w_{L} \geq 0.13$, $1.34 \mathrm{GeV}$ corresponding to $\xi_{Y}=10^{-1}, 10^{-2}$, respectively.

The contributions to the decay $\mu \rightarrow e \gamma$ by virtual charged Higgs $\mathcal{H}_{1,2}^{ \pm(q+1)}$ exchanges are extremely small, compared to those by $Y_{1,2}^{ \pm(q+1)}$ gauge bosons, if one uses the same values of the model's parameters involved in the process. The branching ratios shown in Fig. 7 are smaller than the gauge

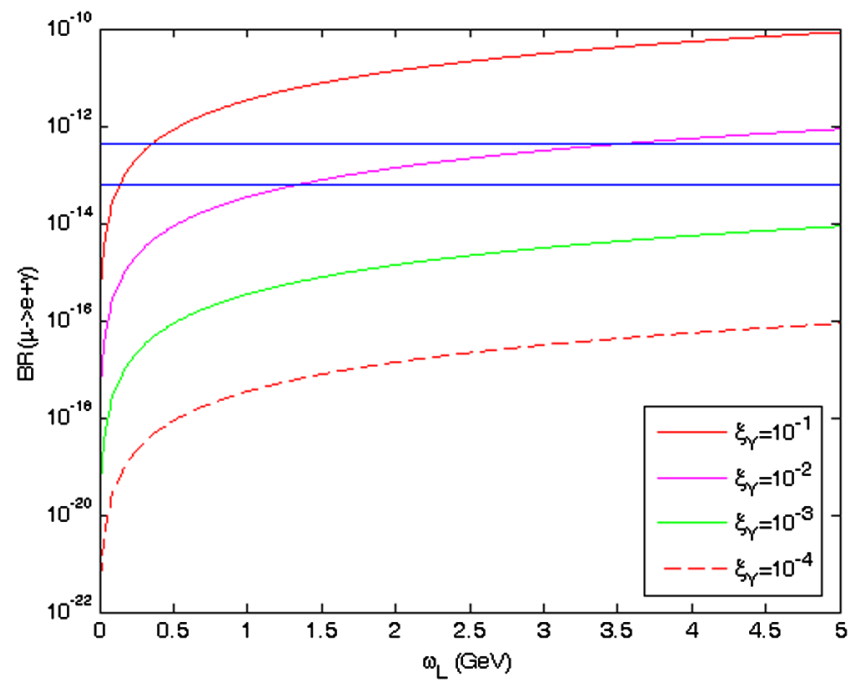

FIG. 6. Dependence of the branching ratio $\operatorname{Br}(\mu \rightarrow e \gamma)$, governed by the virtual $Y_{1,2}^{ \pm(q+1)}$ gauge boson exchanges, on $w_{L}$ for different values of the mixing angle $\xi_{Y}$. The upper and lower lines correspond to the MEG current bound and the near-future sensitivity limit. 


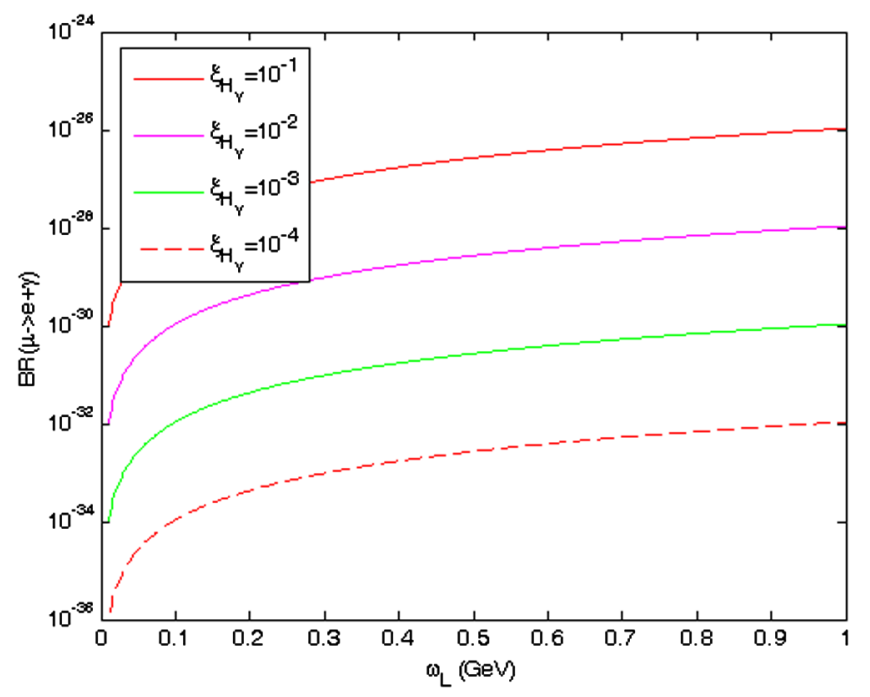

FIG. 7. Dependence of the branching ratio $\operatorname{Br}(\mu \rightarrow e \gamma)$, governed by the virtual charged Higgs $\mathcal{H}_{1,2}^{ \pm(q+1)}$ exchanges, as a function of $w_{L}$ for different values of the mixing angle $\xi_{H_{Y}}$, where we fixed the Higgs masses $M_{H}=10 \mathrm{TeV}$.

ones by 14 orders of magnitude, which are about 13 orders of magnitude below the future MEG sensitivity. It is not hard to see that the branching ratios are strongly suppressed by the ordinary charged-lepton Yukawa couplings $y^{4}$, where the biggest element is only $y_{3} \sim 10^{-2}$, which are much smaller than the gauge contribution.

\section{Other charged LFV processes}

In this model, the charged LFV processes such as $\mu \rightarrow$ $3 e$ and $\tau \rightarrow 3 \mu(3 e)$ can exist at the tree level, exchanged by the charged Higgs $H_{1,2}^{ \pm \pm}$. The $\mu \rightarrow 3 e$ branching ratio in the present scheme with a low scale of new physics of order $10-100 \mathrm{TeV}$ is expected to be in the sensitive ranges of the current and near-future experiments. The present upper bounds on branching ratios of $\tau \rightarrow 3 \mu(3 e)$ are in the order of $10^{-8}$ [15], which are four orders bigger than those of $\mu \rightarrow 3 e$ decay at $10^{-12}$ [15]. Moreover, the $\mu \rightarrow 3 e$ experiment at Paul Scherrer Institute (PSI) is expected to determine the signal of $\operatorname{Br}(\mu \rightarrow 3 e) \geq 10^{-15}$, and its upgrade is sensitive to the $\mu \rightarrow 3 e$ branching ratio not smaller than $10^{-16}$ [24]. Therefore, we need to consider only the process of $\mu \rightarrow 3 e$ decay in which we are concerned with this search.

It is easily verified that, in contrast to the previously mentioned processes, the charged-LFV neutral-Higgs decays, e.g., $h \rightarrow \mu \tau$, receive only one-loop contributions. On the theoretical side, they are strictly suppressed by the heavy particle masses and the loop factor $1 / 16 \pi^{2}$. It is easily proved that such processes satisfy all the current bounds with the chosen parameter regime, since such experimental bounds are less tight [15].
Using the relevant LFV vertices given in Table I, while keeping in mind that the doubly charged Higgs bosons that dominantly contribute to the $\mu \rightarrow 3 e$ decay have the transferred momenta much smaller than their masses, one can write down the effective Lagrangian as

$$
\begin{aligned}
\mathcal{L}_{\text {eff }}(\mu \rightarrow 3 e)= & g_{L S}^{L L}\left(\overline{e_{L}^{c}} \mu_{L}\right)\left(\overline{e_{L}^{c}} e_{L}\right)+g_{R S}^{R R}\left(\overline{e_{R}^{c}} \mu_{R}\right)\left(\overline{e_{R}^{c}} e_{R}\right) \\
& +g_{L S}^{L R}\left(\overline{e_{L}^{c}} \mu_{L}\right)\left(\overline{e_{R}^{c}} e_{R}\right)+g_{R S}^{R L}\left(\overline{e_{R}^{c}} \mu_{R}\right)\left(\overline{e_{L}^{c}} e_{L}\right) .
\end{aligned}
$$

Here, we denote $M_{H_{i}}(i=1,2)$ to be the masses of doubly charged Higgs bosons and

$$
\begin{aligned}
& g_{L S}^{L L}=-\sum_{i=1}^{2} \frac{2}{\left(M_{H_{i}}\right)^{2}}\left(y_{H_{i}}^{L}\right)_{e \mu}\left(y_{H_{i}}^{L}\right)_{e e} \\
& g_{R S}^{R R}=-\sum_{i=1}^{2} \frac{2}{\left(M_{H_{i}}\right)^{2}}\left(y_{H_{i}}^{R}\right)_{e \mu}\left(y_{H_{i}}^{R}\right)_{e e} \\
& g_{L S}^{L R}=-\sum_{i=1}^{2} \frac{1}{\left(M_{H_{i}}\right)^{2}}\left(y_{H_{i}}^{L}\right)_{e \mu}\left(y_{H_{i}}^{R}\right)_{e e} \\
& g_{R S}^{R L}=-\sum_{i=1}^{2} \frac{1}{\left(M_{H_{i}}\right)^{2}}\left(y_{H_{i}}^{R}\right)_{e \mu}\left(y_{H_{i}}^{L}\right)_{e e}
\end{aligned}
$$

The branching ratio is straightforwardly obtained as [25]

$\operatorname{Br}(\mu \rightarrow 3 e)=\frac{1}{32 G_{F}^{2}}\left(\left|g_{L S}^{L L}\right|^{2}+\left|g_{R S}^{R R}\right|^{2}+\left|g_{L S}^{L R}\right|^{2}+\left|g_{R S}^{R L}\right|^{2}\right)$,

where $G_{F}=1.166 \times 10^{-5} \mathrm{GeV}^{2}$ is the Fermi coupling constant.

For numerical evaluation, without loss of generality, we assume that both of the doubly charged Higgs bosons have the same mass; thus, $M_{H_{i}}=M_{H}(i=1,2)$. It is not necessary to consider the mixing angle, $\xi_{H}$, between $\mathrm{H}_{1}^{++}$and $\mathrm{H}_{2}^{++}$for the process in which we are currently interested. Because of the $\xi_{H}$ smallness $\left(\xi_{H} \ll 1\right)$, the dominated contributions come from the terms involving $2\left(y_{H_{1}}^{L}\right)_{e \mu}\left(y_{H_{1}}^{L}\right)_{e e} /\left(M_{H_{1}}\right)^{2}$ and $2\left(y_{H_{2}}^{R}\right)_{e \mu}\left(y_{H_{2}}^{R}\right)_{e e} /\left(M_{H_{2}}\right)^{2}$, which are easily realized as proportional to $\cos \xi_{H}^{2} \simeq 1$, whereas the others are suppressed by a factor either $\sin \xi_{H}$ or $\sin \xi_{H}{ }^{2}$. In the following discussion, we take $\xi_{H}=0.1$. The branching ratio expressed in Eq. (86) is, in fact, inversely proportional to $M_{H}^{4} \Lambda_{R}^{4}$ because it is proportional to $Y_{H_{1,2}}^{R, L} \sim x$, where $x=-U_{R}^{*} M_{R}^{\text {diag }} U_{R}^{\dagger} /\left(\sqrt{2} \Lambda_{R}\right), \quad U_{R}=$ $U_{R}\left(\theta_{12}^{\prime}, \theta_{13}^{\prime}, \theta_{23}^{\prime}, \delta^{\prime}\right)$, and $g_{L(R) S}^{L(R) L(R)} \sim 1 / M_{H}^{2}$. Therefore, the ratio will be strongly suppressed in the large ranges of $\Lambda_{R}$ and $M_{H}$. Taking $M_{R}^{\text {diag }}=(10,20,30) \mathrm{TeV}$ as chosen before, while varying $\theta_{12}^{\prime}, \theta_{13}^{\prime}$, and $\theta_{23}^{\prime}$ in the range of $[0, \pi / 2]$ and $\delta^{\prime}$ in $[0,2 \pi]$, one gets a bound, 




FIG. 8. Branching ratio $\operatorname{Br}(\mu \rightarrow 3 e)$ as a function of doubly charged Higgs masses. The three blue lines, $\operatorname{Br}(\mu \rightarrow 3 e)=$ $10^{-12}, 10^{-15}, 10^{-16}$, correspond to the current experimental upper bound and the sensitivities of PSI and PSI upgraded experiments, respectively.

$0 \leq \operatorname{Br}(\mu \rightarrow 3 e) \leq 1.64 \times 10^{6}\left[\frac{1 \mathrm{TeV}}{M_{H}}\right]^{4}\left[\frac{1 \mathrm{TeV}}{\Lambda_{R}}\right]^{4}$

As a specific case, taking $\theta_{12}^{\prime}=\theta_{13}^{\prime}=\theta_{23}^{\prime}=\pi / 4$ and $\delta^{\prime}=0$, Fig. 8 describes the behavior of the $\mu \rightarrow 3 e$ branching ratio as a function of the doubly charged Higgs masses. The figure reveals a line of a monotonically decreasing function as increasing of $M_{H}$, which is consistent with the fact that the branching ratio is inversely proportional to $M_{H}^{4}$, mentioned above. The lower bounds of the doubly charged Higgs masses corresponding to the current limit, PSI experiment, and its upgraded sensitivities are 14, 79, and $143 \mathrm{TeV}$, respectively. Thus, we apparently conclude that the future PSI experiment is more sensitive to the new physics of the considering model than the MEG, which gives the lower bound $M_{H}=53 \mathrm{TeV}$ for the case $\xi_{H}=0.1$.

\section{CONCLUSION}

When a gauge symmetry is flipped, it leads to a deeper structure that defines a more fundamental theory. For instance, $S U(2)_{L}$ flipped yields electroweak unification; $S U$ (5) flipped defines a seesaw mechanism; and $S O(10)$ flipped leads to $E_{6}$ and promising superstring theories. In this work, we have addressed such a nontrivial task, the flipped trinification and its novel consequences. First of all, a trinification flipped unifies both the 3-3-1 and left-right symmetries. Consequently, this flipped trinification resolves the generation number and the weak parity violation. Additionally, it generates neutrino masses and dark matter naturally via the gauge symmetry.

An important feature of the flipped trinification is that it presents the flavor-changing currents in both quark and lepton sectors. We have probed that the quark FCNCs bound the new physics scale to be at or beyond several tens of teraelectron-volts via the neutral meson mixings, $B_{d, s}^{0}-\bar{B}_{d, s}^{0}$. The charged LFV via the decay $\mu \rightarrow e \gamma$ yield mostly the same bound, whereas the other processes such as $\tau \rightarrow 3 \mu(3 e)$ and $h \rightarrow \mu \tau$ are easily experimentally satisfied. The process $\mu \rightarrow$ $3 e$ receives tree-level contributions by the doubly charged Higgs bosons and presents the same limit on the new physics as the meson mixing and $\mu \rightarrow e \gamma$ do.

All the results indicate that the trinification is possibly flipped at tens of tera-electron-volts. Additionally, the contributions of the new particles other than the left-right symmetric model are important to set the charged LFV and quark FCNC observables, which can be used to prove or rule out this proposal.

\section{ACKNOWLEDGMENTS}

This research is funded by Vietnam National Foundation for Science and Technology Development under Grant No. 103.01-2014.89.

\section{APPENDIX: NEUTRAL GAUGE BOSON MASS MATRICES}

For convenience in reading, in this Appendix, we supply the full neutral gauge boson mass matrix as well as the $3 \times 3$ mass matrix of new neutral gauge bosons.

After the symmetry breaking, the neutral gauge bosons $\left(A_{3 L}, A_{3 R}, A_{8 L}, A_{8 R}\right.$, and $\left.B\right)$ in such order possess a mass matrix,

$$
\frac{g_{L}^{2}}{4}\left(\begin{array}{ccccc}
u_{1}^{2}+u_{2}^{2}+4 \Lambda_{L}^{2} & -t_{R}\left(u_{1}^{2}+u_{2}^{2}\right) & \frac{u_{1}^{2}-u_{2}^{2}+4 \Lambda_{L}^{2}}{\sqrt{3}} & \frac{t_{R}\left(u_{2}^{2}-u_{1}^{2}\right)}{\sqrt{3}} & m_{15} \\
-t_{R}\left(u_{1}^{2}+u_{2}^{2}\right) & t_{R}^{2}\left(u_{1}^{2}+u_{2}^{2}+4 \Lambda_{R}^{2}\right) & \frac{t_{R}\left(u_{2}^{2}-u_{1}^{2}\right)}{\sqrt{3}} & \frac{t_{R}^{2}\left(u_{1}^{2}-u_{2}^{2}+4 \Lambda_{R}^{2}\right)}{\sqrt{3}} & m_{25} \\
\frac{u_{1}^{2}-u_{2}^{2}+4 \Lambda_{L}^{2}}{\sqrt{3}} & \frac{t_{R}\left(u_{2}^{2}-u_{1}^{2}\right)}{\sqrt{3}} & m_{33} & -\frac{1}{3} t_{R}\left(u_{1}^{2}+u_{2}^{2}+4 w^{2}\right) & m_{35} \\
\frac{t_{R}\left(u_{2}^{2}-u_{1}^{2}\right)}{\sqrt{3}} & \frac{t_{R}^{2}\left(u_{1}^{2}-u_{2}^{2}+4 \Lambda_{R}^{2}\right)}{\sqrt{3}} & -\frac{1}{3} t_{R}\left(u_{1}^{2}+u_{2}^{2}+4 w^{2}\right) & m_{44} & m_{45} \\
m_{15} & m_{25} & m_{35} & m_{45} & m_{55}
\end{array}\right),
$$


where we define for short

$$
\begin{gathered}
m_{15}=-\frac{4 t_{X}(3+\sqrt{3} \beta) \Lambda_{L}^{2}}{3}, \quad m_{25}=-\frac{4 t_{R} t_{X} \sqrt{3}(\sqrt{3}+\beta) \Lambda_{R}^{2}}{3}, \\
m_{35}=-\frac{4 t_{X}\left(w_{L}^{2} \beta+(\sqrt{3}+\beta) \Lambda_{L}^{2}\right)}{3}, \quad m_{45}=-\frac{4 t_{R} t_{X}\left(w_{R}^{2} \beta+(\sqrt{3}+\beta) \Lambda_{R}^{2}\right)}{3}, \\
m_{55}=\frac{4 t_{X}^{2}\left(\beta^{2}\left(w_{L}^{2}+w_{R}^{2}\right)+(\sqrt{3}+\beta)^{2}\left(\Lambda_{L}+\Lambda_{R}^{2}\right)\right)}{3}, \\
m_{33}=\frac{u_{1}^{2}+u_{2}^{2}+4\left(w^{2}+w_{L}^{2}+\Lambda_{L}^{2}\right)}{3}, \quad m_{44}=\frac{t_{R}^{2}\left(u_{1}^{2}+u_{2}^{2}+4\left(w^{2}+w_{R}^{2}+\Lambda_{R}^{2}\right)\right)}{3} .
\end{gathered}
$$

Changing to the new basis $\left(A, Z_{L}, Z_{L}^{\prime}, Z_{R}, Z_{R}^{\prime}\right)$, we obtain $A$ and $Z_{L}$ identical to the standard model photon and $Z$ boson, respectively, which are light and decoupled. The remaining fields $\left(Z_{L}^{\prime}, Z_{R}\right.$, and $\left.Z_{R}^{\prime}\right)$ are new and mix via a $3 \times 3$ mass matrix,

$$
M_{3 \times 3}=\frac{g_{L}^{2}}{4}\left(\begin{array}{ccc}
m_{11}^{\prime} & m_{12}^{\prime} & m_{13}^{\prime} \\
m_{21}^{\prime} & m_{22}^{\prime} & m_{23}^{\prime} \\
m_{13}^{\prime} & m_{23}^{\prime} & m_{33}^{\prime}
\end{array}\right),
$$

where $m_{i j}$ are defined as

$$
\begin{aligned}
& m_{11}^{\prime}=\frac{4 c_{W}^{2} w^{2}}{3\left(c_{W}^{2}-s_{W}^{2} \beta^{2}\right)}, \\
& m_{12}^{\prime}=\frac{4 t_{R} w^{2} c_{W} s_{W}^{2}}{3\left(c_{W}^{2}-s_{W}^{2} \beta^{2}\right) \sqrt{t_{R}^{2}-\left(1+t_{R}^{2}\right)\left(1+\beta^{2}\right) s_{W}^{2}} \sqrt{t_{R}^{2}\left(1+\frac{\beta^{2} s_{W}^{2}}{t_{R}^{2}-\left(1+t_{R}^{2}\right)\left(1+\beta^{2}\right) s_{W}^{2}}\right)}}, \\
& m_{13}^{\prime}=\frac{4 \sqrt{2} t_{R}^{2} w^{2} c_{W}}{3 \sqrt{-1+t_{R}^{2}-\left(1+t_{R}^{2}\right)\left(\beta^{2}-\left(1+\beta^{2}\right) c_{2}\right)}\left(1+\frac{\beta^{2} s_{W}^{2}}{t_{R}^{2}-\left(1+t_{R}^{2}\right)\left(1+\beta^{2}\right) s_{W}^{2}}\right)\left(1+\frac{\left(1+\beta^{2}\right) s_{W}^{2}}{t_{R}^{2}-\left(1+t_{R}^{2}\right)\left(1+\beta^{2}\right) s_{W}^{2}}\right)}, \\
& m_{22}^{\prime}=\frac{4\left(3 t_{R}^{4} \Lambda_{R}^{2}-6 t_{R}^{4}\left(1+\beta^{2}\right) \Lambda_{R}^{2} s_{W}^{2}+\left(\beta^{2} w^{2}+3 t_{R}^{4}\left(1+\beta^{2}\right)^{2} \Lambda_{R}^{2}\right) s_{W}^{4}\right)}{3\left(\left(1+\beta^{2}\right) s_{W}^{2}-1\right)\left(-t_{R}^{2}+\left(1+t_{R}^{2}\left(1+\beta^{2}\right)\right) s_{W}^{2}\right)}, \\
& m_{23}^{\prime}=\frac{-4 t_{R}^{2} w^{2} \beta\left(1+\beta^{2}\right)-4 t_{R}^{4}\left(\beta w^{2}+(\sqrt{3}-3 \beta) \Lambda_{R}^{2}\right)\left(1+\beta^{2}-c_{W}^{-2}\right)-4 \sqrt{3} t_{R}^{6} \Lambda_{R}^{2}\left(1+\beta^{2}-c_{W}^{-2}\right)^{2}}{3\left(1+t_{R}^{2}\left(1+\beta^{2}\right)-t_{R}^{2} c_{W}^{-2}\right)\left(\left(1+t_{R}^{2}\right)\left(1+\beta^{2}\right)-t_{R}^{2} c_{W}^{-2}\right) \sqrt{\frac{2+\beta^{2}}{\left(1+\beta^{2}\right)\left(-t_{R}^{2}-1\right)+t_{R}^{2} c_{W}^{2}}}}, \\
& m_{33}=\frac{4 t_{R}^{2}\left(\left(w^{2}+\Lambda_{R}^{2}+w_{R}^{2}\right)\left(t_{R}^{2}-\left(1+t_{R}^{2}\right)\left(1+\beta^{2}\right) s_{W}^{2}\right)^{2}+\mathcal{M}_{33}\right)}{3\left(t_{R}^{2}-\left(1+t_{R}^{2}\right)\left(1+\beta^{2}\right) s_{W}^{2}+\beta^{2} s_{W}^{2}\right)},
\end{aligned}
$$

where $\mathcal{M}_{33}$ takes the form

$$
\begin{aligned}
\mathcal{M}_{33}= & 2 t_{R}^{2} \beta\left((\sqrt{3}+\beta) \Lambda_{R}^{2}+\beta w_{R}^{2}\right) s_{W}^{2}+\beta\left(\left(-2 \sqrt{3}+\beta-\beta^{3}-2 t_{R}^{2}(\sqrt{3}+\beta)\left(1+\beta^{2}\right)\right) \Lambda_{R}^{2}\right) s_{W}^{4} \\
& -\beta s_{W}^{4}\left(2+\beta^{2}+2 t_{R}^{2}\left(1+\beta^{2}\right)\right) w_{R}^{2} .
\end{aligned}
$$


[1] T. Kajita, Rev. Mod. Phys. 88, 030501 (2016); A. B. McDonald, Rev. Mod. Phys. 88, 030502 (2016).

[2] P. Minkowski, Phys. Lett. B 67, 421 (1977); M. Gell-Mann, P. Ramond, and R. Slansky, Complex Spinors and Unified Theories, in Supergravity, edited by P. van Nieuwenhuizen and D.Z. Freedman (North Holland, Amsterdam, 1979), p. 315; T. Yanagida, in Proceedings of the Workshop on the Unified Theory and the Baryon Number in the Universe, edited by O. Sawada and A. Sugamoto (KEK, Tsukuba, Japan, 1979), p. 95; S. L. Glashow, The future of elementary particle physics, in Proceedings of the 1979 Cargèse Summer Institute on Quarks and Leptons, edited by M. Lévy et al. (Plenum, New York, 1980), pp. 687-713; R. N. Mohapatra and G. Senjanović, Phys. Rev. Lett. 44, 912 (1980); Phys. Rev. D 23, 165 (1981); G. Lazarides, Q. Shafi, and C. Wetterich, Nucl. Phys. B181, 287 (1981); J. Schechter and J. W. F. Valle, Phys. Rev. D 22, 2227 (1980); 25, 774 (1982).

[3] J. C. Pati and A. Salam, Phys. Rev. D 10, 275 (1974); R. N. Mohapatra and J. C. Pati, Phys. Rev. D 11, 566 (1975); 11, 2558 (1975); G. Senjanović and R. N. Mohapatra, Phys. Rev. D 12, 1502 (1975); G. Senjanović, Nucl. Phys. B153, 334 (1979); R. N. Mohapatra and G. Senjanović, Phys. Rev. D 23, 165 (1981).

[4] D. N. Spergel et al. (WMAP Collaboration), Astrophys. J. Suppl. Ser. 170, 377 (2007); P. A. R. Ade et al. (Planck Collaboration), Astron. Astrophys. 571, A1 (2014); see, for reviews, G. Bertone, D. Hooper, and J. Silk, Phys. Rep. 405, 279 (2005); G. Jungman, M. Kamionkowski, and K. Griest, Phys. Rep. 267, 195 (1996).

[5] F. Bezrukov, H. Hettmansperger, and M. Lindner, Phys. Rev. D 81, 085032 (2010); M. Nemevsek, G. Senjanovic, and Y. Zhang, J. Cosmol. Astropart. Phys. 07 (2012) 006; J. Barry, J. Heeck, and W. Rodejohann, J. High Energy Phys. 07 (2014) 081.

[6] J. Heeck and S. Patra, Phys. Rev. Lett. 115, 121804 (2015); C. Garcia-Cely and J. Heeck, J. Cosmol. Astropart. Phys. 03 (2016) 021; M. Cirelli, N. Fornengo, and A. Strumia, Nucl. Phys. B753, 178 (2006); A. Berlin, P. J. Fox, D. Hooper, and G. Mohlabeng, J. Cosmol. Astropart. Phys. 06 (2016) 016; P. S. B. Dev, R. N. Mohapatra, and Y. Zhang, J. High Energy Phys. 11 (2016) 077.

[7] F. Pisano and V. Pleitez, Phys. Rev. D 46, 410 (1992); P. H. Frampton, Phys. Rev. Lett. 69, 2889 (1992); R. Foot, O. F. Hernandez, F. Pisano, and V. Pleitez, Phys. Rev. D 47, 4158 (1993); M. Singer, J. W. F. Valle, and J. Schechter, Phys. Rev. D 22, 738 (1980); J. C. Montero, F. Pisano, and V. Pleitez, Phys. Rev. D 47, 2918 (1993); R. Foot, H. N. Long, and T. A. Tran, Phys. Rev. D 50, R34 (1994); P. V. Dong, H. N. Long, D. T. Nhung, and D. V. Soa, Phys. Rev. D 73, 035004 (2006); S. M. Boucenna, J. W. F. Valle, and A. Vicente, Phys. Rev. D 92, 053001 (2015); J. W. F. Valle and C. A. Vaquera-Araujo, Phys. Lett. B 755, 363 (2016).

[8] P. V. Dong, T. D. Tham, and H. T. Hung, Phys. Rev. D 87, 115003 (2013); P. V. Dong, D. T. Huong, F. S. Queiroz, and N. T. Thuy, Phys. Rev. D 90, 075021 (2014); D. T. Huong,
P. V. Dong, C. S. Kim, and N. T. Thuy, Phys. Rev. D 91, 055023 (2015); P. V. Dong, Phys. Rev. D 92, 055026 (2015); P. V. Dong and D. T. Si, Phys. Rev. D 93, 115003 (2016); A. Alves, G. Arcadi, P. V. Dong, L. Duarte, F. S. Queiroz, and J. W. F. Valle, Phys. Lett. B 772, 825 (2017); D. T. Huong and P. V. Dong, Eur. Phys. J. C 77, 204 (2017).

[9] P. V. Dong, D. T. Huong, F. S. Queiroz, J. W. F. Valle, and C. A. Vaquera-Araujo, J. High Energy Phys. 04 (2018) 143.

[10] A. G. Dias, C. A. de S. Pires, and P. S. Rodrigues da Silva, Phys. Rev. D 82, 035013 (2010); C. P. Ferreira, M. M. Guzzo, and P. C. de Holanda, Braz. J. Phys. 46, 453 (2016); D. T. Huong and P. V. Dong, Phys. Rev. D 93, 095019 (2016); P. V. Dong and D. T. Huong, Commun. Phys. 28, 21 (2018); M. Reig, J. W. F. Valle, and C. A. Vaquera-Araujo, Phys. Lett. B 766, 35 (2017); J. High Energy Phys. 05 (2017) 100; C. Hati, S. Patra, M. Reig, J. W. F. Valle, and C. A. Vaquera-Araujo, Phys. Rev. D 96, 015004 (2017); P. V. Dong, D. T. Huong, D. V. Loi, N. T. Nhuan, and N. T. K. Ngan, Phys. Rev. D 95, 075034 (2017); C. Kownacki, E. Ma, N. Pollard, O. Popov, and M. Zakeri, Phys. Lett. B 777, 121 (2018); E. Ma, Phys. Lett. B 780, 533 (2018); C. Kownacki, E. Ma, N. Pollard, O. Popov, and M. Zakeri, Nucl. Phys. B928, 520 (2018).

[11] Y. Achiman and B. Stech, in Advanced Summer Institute on New Phenomena in Lepton and Hadron Physics, edited by D. E. C. Fries and J. Wess (Plenum, New York, 1979), p. 303; A. De Rujula, H. Georgi, and S. L. Glashow, in Fifth Workshop on Grand Unification, edited by K. Kang, H. Fried, and P. Frampton (World Scientific, Singapore, 1984), p. 88.

[12] P. V. Dong and H. N. Long, Eur. Phys. J. C 42, 325 (2005).

[13] F. Gabbiani, E. Gabrielli, A. Masiero, and L. Silvestrini, Nucl. Phys. B477, 321 (1996).

[14] T. Jubb, M. Kirk, A. Lenz, and G. Tetlalmatzi-Xolocotzi, Nucl. Phys. B915, 431 (2017); A. J. Buras and F. D. Fazio, J. High Energy Phys. 08 (2016) 115.

[15] M. Tanabashi et al. (Particle Data Group), Phys. Rev. D 98, 030001 (2018).

[16] S. T. Petcov, Yad. Fiz. 25, 641 (1977) [Sov. J. Nucl. Phys. 25, 340 (1977)]; Yad. Fiz. 25, 1336(E) (1977) [Sov. J. Nucl. Phys. 25, 698(E) (1977)].

[17] S. M. Bilenky, S. T. Petcov, and B. Pontecorvo, Phys. Lett. B 67B, 309 (1977).

[18] T. P. Cheng and L. F. Li, Phys. Rev. Lett. 45, 1908 (1980).

[19] E. Ma, M. Raidal, and U. Sarkar, Nucl. Phys. B615, 313 (2001).

[20] J. Bernabeu, A. Pich, and A. Santamaria, Z. Phys. C 30, 213 (1986).

[21] G. K. Leontaris, K. Tamvakis, and J. D. Vergados, Phys. Lett. 162B, 153 (1985).

[22] D. N. Dinh, A. Ibarra, E. Molinaro, and S. T. Petcov, J. High Energy Phys. 08 (2012) 125; 09 (2013) 23.

[23] D. T. Huong and P. V. Dong, Eur. Phys. J. C 77, 204 (2017).

[24] A. Blondel et al., arXiv:1301.6113.

[25] T. Goto, Y. Okada, and Y. Yamamoto, Phys. Rev. D 83, 053011 (2011). 